\title{
Synthesis, crystal and topological electronic structures of new bismuth tellurohalides $\mathrm{Bi}_{2} \mathrm{TeBr}$ and $\mathrm{Bi}_{3} \mathrm{TeBr}$
}

\author{
Alexander Zeugner ${ }^{1}$, Johannes Teichert', Martin Kaiser ${ }^{1}$, Tatiana V. Menshchikova ${ }^{2}$, \\ Igor P. Rusinov ${ }^{2,3}$, Anton V. Markelov ${ }^{4}$, Evgueni V. Chulkov ${ }^{2,3,5,6,7}$, Thomas Doert ${ }^{1}$, Michael Ruck', \\ Anna Isaeva ${ }^{*}$
}

1 Technische Universität Dresden, Faculty of Chemistry and Food Chemistry, Helmholtzstraße 10, o1069 Dresden, Germany 2 Tomsk State University, pr. Lenina, 36, 634050 Tomsk, Russia

3 St. Petersburg State University, Universitetskaya nab., 7/9, 199034 St. Petersburg, Russia 4 SuperOx, Odintsovskii district, 143082 Moscow region, Russia

5 Donostia International Physics Center (DIPC), Paseo de Manuel Lardizabal, 4, 20018 San Sebastian/Donostia, Basque Country, Spain

6 Departamento de Fisica de Materiales, Facultad de Ciencias Quimicas, UPV/EHU, 20o8o San Sebastian/Donostia, Basque Country, Spain

7 Centro de Fisica de Materiales CFM-MPC, Centro Mixto CSIC-UPV/EHU, 2oo8o San Sebastian/Donostia, Basque Country, Spain

\begin{abstract}
Halogen substitution, i. e. bromine for iodine, in the series of topological $\mathrm{Bi}_{n} \mathrm{TeI}(n=1,2,3)$ materials is conducted in order to explore an impact of anion exchange on the topological electronic structure. In the proof-of-concept study we demonstrate the applicability of the modular view on the crystal and electronic structures of the new $\mathrm{Bi}_{2} \mathrm{TeBr}$ and $\mathrm{Bi}_{3} \mathrm{TeBr}$ compounds. Along with the isostructural telluroiodides, they constitute a family of layered structures that are stacked from two basic building modules, ${ }_{\infty}^{2}\left[\mathrm{Bi}_{2}\right]$ and ${ }_{\infty}^{2}[\mathrm{BiTe} X](X=\mathrm{I}, \mathrm{Br})$. We present solid-state synthesis, thermochemical studies, crystal growth and crystal-structure elucidation of $\mathrm{Bi}_{2} \mathrm{TeBr}$ (sp.gr. $R \overline{3} m$ (no. 166), $a=433.04(2) \mathrm{pm}, c=$ $5081.6(3) \mathrm{pm}$ ) and $\mathrm{Bi}_{3} \mathrm{TeBr}$ (sp.gr. R3m (no. 16o), $a=437.68(3) \mathrm{pm}, c=3122.9(3) \mathrm{pm}$ ). First-principles calculations establish the topological nature of $\mathrm{Bi}_{2} \mathrm{TeBr}$ and $\mathrm{Bi}_{3} \mathrm{TeBr}$. General aspects of chemical bonding appear to be similar for $\mathrm{Bi} \mathrm{i}_{n} \mathrm{Te} X(X=\mathrm{I}$, $\mathrm{Br}$ ) with the same $n$, so that the alternation of the global gap size upon substitution is insignificant. The complex topological inversion proceeds between the states of two distinct modules, ${ }_{\infty}^{2}\left[\mathrm{Bi}_{2}\right]$ and ${ }_{\infty}^{2}[\mathrm{BiTeBr}$; thus, the title compounds can be seen as heterostructures built via a modular principle. Furthermore, highly disordered as well as incommensurately modulated ternary phase(s) are documented near the $\mathrm{Bi}_{2} \mathrm{TeBr}$ composition. Single-crystal X-ray diffraction experiments on $\mathrm{BiTeBr}$ and $\mathrm{Bi}_{2} \mathrm{TeI}$ resolve some discrepancies in the prior published work.
\end{abstract}

\section{INTRODUCTION}

Bismuth tellurohalides $\operatorname{BiTe} X(X=\mathrm{Cl}, \mathrm{Br}, \mathrm{I})$ constitute a multi-purpose material platform offering an attractive combination of functionalities such as fair thermoelectric performance, ${ }^{1,2}$ giant Rashba splitting of bulk and surface bands due to strong spin-orbit coupling and a lack of inversion symmetry ${ }^{3-10}$, ambipolar conduction on different surface terminations thanks to the polarity of the structural fragments and strong internal electric fields, ${ }^{11-13}$ pressure-induced quantum topological phase transitions and lattice dynamics ${ }^{14-23}$ as well as, possibly, topological superconductivity $^{24-26}$. An interplay of these qualities enhances relevance of $\mathrm{BiTe} X$ for future applications in spintronics near room temperature and creates more space for novel phenomena.

Chemical modification of structure and composition is a versatile tool to map out rich phase diagrams of possible electronic and magnetic ground states. There are several examples how physical properties of $\mathrm{BiTe} X$ can be varied by chemical means.

Improved thermoelectric performance was reported for a $\mathrm{Cu}$-intercalated derivative ${ }^{27}$ of $\mathrm{BiTeI}$ and $\mathrm{Br}$-substituted $\mathrm{BiTeI}_{1-x} \mathrm{Br}_{x}(x=0.06,0.12)^{28}$. Higher $\mathrm{Br}$ doping rates have not been investigated. Pure BiTeBr has a twice higher ZT value near room temperature than BiTeI. ${ }^{11}$ Moreover, the TE figure-of-merit decreased in polycrystalline CuI/BiTeI, 
$\mathrm{BiI}_{3} / \mathrm{BiTeI}$ and Bi/BiTeI composites. ${ }^{2,29}$ Magnetic doping of BiTeI by $\mathrm{Mn}$ and $\mathrm{V}$ has also been attempted. ${ }^{30}$ Bulk BizTeI has a low ZT value that can be moderately improved by Zn doping. ${ }^{31}$

The layered BiTeI structure type can be altered in a more complex way as it incorporates corrugated ${ }_{\infty}^{2}\left[\mathrm{Bi}_{2}\right]$ layers into the van der Waals gaps and, thus, transforms into $\mathrm{Bi}_{2} \mathrm{TeI}=\left[\mathrm{Bi}_{2}\right] \cdot 2[\mathrm{BiTeI}]^{32,33}$ or $\mathrm{Bi}_{3} \mathrm{TeI}=\left[\mathrm{Bi}_{2}\right] \cdot[\mathrm{BiTeI}]^{33}$ structure types. Remarkably, these heterostructures are thermodynamically stable and can be synthesized as phase-pure powders and single-crystals. ${ }^{33}$

Furthermore, this structural modification augments the topological properties of the resultant lattices. The first building module, ${ }_{\infty}^{2}\left[\mathrm{Bi}_{2}\right]$ (also referred to as Bi-bilayer ${ }^{34}$ ), is a two-dimensional topological insulator (2D TI). ${ }^{35,36}$ Elemental bismuth can be regarded as a stack of interacting Bi-bilayers. Hybridization of the states along the stacking direction may result in $3 \mathrm{D}$ topological properties, although there is so far no consent whether pure bismuth has a nontrivial topology. ${ }^{37-41}$ A rough estimation using the bond-length bond-strength correlation assigns about $20 \%$ of bonding of every $\mathrm{Bi}$ atom in the structure of elemental bismuth to interlayer bonding. Both a single [BiTeX] layer and the periodic $B i T e X$ lattice are topologically trivial under ambient conditions. Hydrostatic pressure may induce a quantum topological transition in bulk BiTeI via a band inversion at the A point of the ${ }_{3} \mathrm{D}$ Brillouin zone (BZ) between the states of all atomic sorts. ${ }^{14,16}$ Chemical bonding between the ${ }_{\infty}^{2}\left[\mathrm{Bi}_{2}\right]$ and ${ }_{\infty}^{2}[\mathrm{BiTeI}]$ modules in $\mathrm{Bi}_{2}$ TeI exceeds the van der Waals interactions in pristine BiTeI, so that a topological inversion is realized $\mathrm{Bi}_{2}$ TeI at the $\mathrm{Z}$ point of the $3 \mathrm{D} \mathrm{BZ}$ under normal pressure. ${ }^{42-44}$ Enhanced hybridization between the states of the Bi-bilayer and the BiTeI matrix results in a dual topological insulator in the case of $\mathrm{Bi}_{2} \mathrm{TeI}$ and in a topological metal in the case of $\mathrm{Bi}_{3} \mathrm{TeI}^{33}$. A similarly developing tendency in topological properties is observed in the $\mathrm{Bi}_{2} \mathrm{Te}_{3}-$ $\mathrm{BiTe}-\mathrm{Bi}_{4} \mathrm{Se}_{3}$ series. $^{33}$

We have recently described the concurrent evolution of structural and topological properties in the BiTeI - $\mathrm{Bi}_{2} \mathrm{TeI}$ - $\mathrm{Bi}_{3} \mathrm{TeI}$ series in terms of a modular principle. ${ }^{33}$ Our main aim is to derive a phenomenological principle that could assist in the pursuit of new topological materials, ${ }^{45}$ so we want to put the modular design to a further test. The structure-property relation cannot be established for topological materials in a straightforward way, since, in general, the topological properties of bulk cannot be reduced to a superposition of the topological properties of its structure modules. Chemical bonding leading to enhanced hybridization and the resulting $k_{\mathrm{z}}$ dispersion cannot be quantified by simple means. Since the classic concept of topological insulators ${ }^{46}$ does not directly include crystallography or chemical bonding, it does not directly point out real materials with complex symmetries and bonding patterns. Attempts to establish such relations have recently started. ${ }^{47}$ The proposed analysis utilizes a local orbital-based picture. Modular design of new materials proceeds on the level of larger structure fragments, i. e. complexes, clusters, chains, layers, etc., and is more feasible for a chemist than on the level of atoms and local point symmetries. Modular organization of solids diversifies the means of control of physical properties, since single modules can be tuned separately. Modulation doping of semiconductor heterostructures and hightemperature superconductors as well as the phonon-glass electron-crystal concept in thermoelectrics illustrate successful applications of this very general idea. ${ }^{4-50}$

Topological bismuth telluroiodides offer two structure modules, ${ }_{\infty}^{2}\left[\mathrm{Bi}_{2}\right]$ and ${ }_{\infty}^{2}[\mathrm{BiTeI}]$, the former being a $2 \mathrm{D} \mathrm{TI}$. Our goal was to examine the applicability of the modular principle to the crystal structure, $i$. e. to expand the $\mathrm{Bi}_{n} \mathrm{TeI}$ structure family via bromine substitution in a selected module. We have synthesized stable $\mathrm{Bi}_{2} \mathrm{TeBr}$ and $\mathrm{Bi}_{3} \mathrm{TeBr}$ compounds and elucidated their crystal structures. Another aim was to explore if the modular principle is pertinent to topological properties. For that, we traced the changes in the electronic spectra of the entire heterostructure by first-principle calculations upon chemical modifications in the selected module. As a result, we present a proof-of-concept study of the modular principle applied to $\mathrm{Bi}_{2} \mathrm{TeBr}$ and $\mathrm{Bi}_{3} \mathrm{TeBr}$ and demonstrate the impact of anion exchange on the crystal structure and topological electronic structure. First-principles calculations indicate that the topological band inversions in $\mathrm{Bi}_{2} \mathrm{TeBr}$ and $\mathrm{Bi}_{3} \mathrm{TeBr}$ take place between the states of two distinct modules. The observed chemical and electronic flexibility let us believe that further new effects could be discovered in bismuth tellurohalides via exertion of external perturbations as well as chemical pressure.

\section{RESULTS AND DISCUSSION}

\section{Synthesis and Thermal Stability of $\mathrm{Bi}_{n} \mathrm{TeBr}$}

Large hexagonal platelets of $\mathrm{BiTeI}$ and $\mathrm{BiTeBr}$ were first obtained in $1951 .{ }^{51}$ Crystal-growth routes ${ }^{52}$ and phase relations in the pseudo-binary $\mathrm{BiBr}_{3}-\mathrm{Bi}_{2} \mathrm{Te}_{3}$ section ${ }^{53,54}$ have been studied in full detail. In contrast to that, syntheses of $\mathrm{Bi}_{2} \mathrm{TeI}^{32,33}$ and $\mathrm{Bi}_{3} \mathrm{TeI}^{33}$ were reported quite recently. We have applied the following core principles, that were determined previously for the $\mathrm{Bi}-\mathrm{Te}-\mathrm{I}$ system, ${ }^{33}$ to the present study of the $\mathrm{Bi}-\mathrm{Te}-\mathrm{Br}$ system:

1. Tribochemical pre-activation of the reactants by ball-milling.

2. Thermochemical analysis of the mechanically activated mixtures.

3. Solid-state synthesis in the sub-solidus temperature region.

The targeted ternary phases have formed in all starting mixtures with the nominal compositions $\mathrm{Bi}_{n} \operatorname{TeBr}(n=1,2$, 3) already after the ball-milling (Fig. S1). No sign of $\mathrm{BiBr}_{3}$ evaporation or decomposition was observed at this stage. The previously unaccounted PXRD patterns of $\mathrm{Bi}_{2} \mathrm{TeBr}$ and $\mathrm{Bi}_{3} \mathrm{TeBr}$ were preliminary indexed by analogy with the respective $\mathrm{Bi}_{n} \mathrm{TeI}^{33}$. The resultant powdered samples were divided in two batches, one to be analyzed by differential scanning calorimetry (DSC) and the other to be consequently annealed in the sub-solidus range. 
Our thermochemical experiments (see Supporting Information for an extended discussion, Fig. S2-S4) show that $\mathrm{Bi}_{2} \mathrm{TeBr}$ and $\mathrm{Bi}_{3} \mathrm{TeBr}$ melt at lower temperatures than $\mathrm{BiTeBr}^{54}$ and by ca. $10{ }^{\circ} \mathrm{C}$ lower than their $\mathrm{Bi}_{n} \mathrm{TeI}$ analogs ${ }^{33}$. We establish incongruent melting of $\mathrm{Bi}_{3} \mathrm{TeBr}$ at around $400{ }^{\circ} \mathrm{C}$, resembling the behavior of $\mathrm{Bi}_{3} \mathrm{Tel}^{33}$. The exact melting behavior of $\mathrm{Bi}_{2} \mathrm{TeBr}$ cannot be conclusively elucidated from the available data. Interpretation is hampered by presence of an "intermediate" phase that exhibits a shifted and broadened set of reflections with respect to $\mathrm{Bi}_{2} \mathrm{TeBr}$ that hints at massive structural disorder or poor crystallinity with small particle or domain size (Fig. S5).

Nevertheless, the DSC results enabled us to make an informed choice of the synthetic temperatures for phasepure samples. The remaining parts of the ball-milled batches were annealed at the temperatures in the subsolidus range, e. g. $385-450^{\circ} \mathrm{C}$ depending on the sample composition. These solid-state reactions yield nearly phase-pure powder samples (Fig. 1) of $\mathrm{Bi}_{n} \mathrm{TeBr}$, whose reflections accord well with the theoretical patterns based on model $\mathrm{Bi}_{n} \mathrm{TeI}$ analogs. The $\mathrm{BiTeBr}$ sample again shows admixtures of $\mathrm{Bi}_{2} \mathrm{Te}_{3}$ and for the sample of the nominal composition " $\mathrm{Bi}_{3} \mathrm{TeBr}$ " a slight bismuth loss is observed that leads to formation of $\mathrm{Bi}_{2} \mathrm{TeBr}$ admixture.

The crucial role of thorough homogenization (the above-listed synthetic principle 1) becomes apparent from the following example. The "intermediate" phase is obtained as the major product from a mixture of $\mathrm{Bi}, \mathrm{BiBr}_{3}$ and $\mathrm{Te}$ with the nominal composition " $\mathrm{Bi}_{2} \mathrm{TeBr}$ " after annealing at $425^{\circ} \mathrm{C}$ for 5 days and quenching (Fig. S6). As opposed to that, a similar mixture subjected to ballmilling and annealed at $400{ }^{\circ} \mathrm{C}$ for 5 days contains phasepure $\mathrm{Bi}_{2} \mathrm{TeBr}$ (Fig. 1). Furthermore, mixtures of $\mathrm{Bi}, \mathrm{BiBr}_{3}$ and $\mathrm{Te}$ with the nominal compositions " $\mathrm{Bi}_{2.5} \mathrm{TeBr}$ " and " $\mathrm{Bi}_{3} \mathrm{TeBr}$ ", that were annealed at $425^{\circ} \mathrm{C}$ for 5 days (i. e. above the melting point of $\mathrm{Bi}_{3} \mathrm{TeBr}$, cf. the principle 1 above) and quenched, contain phase mixtures of $\mathrm{Bi}_{3} \mathrm{TeBr}$, $\mathrm{Bi}_{2} \mathrm{TeBr}$ and $\mathrm{Bi}$.

Similar to $\mathrm{Bi}_{n} \mathrm{TeI}^{33}$, we did not find any Bi-richer ternaries or an extended homogeneity range on the bismuthrich side. An annealed sample with the nominal composition " $\mathrm{Bi}_{4} \mathrm{TeBr}$ " comprises a mixture of $\mathrm{Bi}_{3} \mathrm{TeBr}$, that does not show any changes in the unit cell parameters, and residual Bi (Fig. S7).

On this stage, a cautious conclusion can be drawn that the new $\mathrm{Bi}_{n} \mathrm{TeBr}(n=2,3)$ are indeed structurally analogous to the telluroiodides (Table 1). No superstructure reflections and no notable deviations from the targeted compositions were observed.

The ED patterns of $\mathrm{BiTeBr}, \mathrm{Bi}_{3} \mathrm{TeBr}$ and $\mathrm{Bi}_{2} \mathrm{TeBr}$ along the stacking direction (Fig. 2) strongly resemble the corresponding $\mathrm{Bi}_{n} \mathrm{TeI}^{33,43}$. The indexing concords with the lattice parameters (Table 1) and trigonal symmetry. The "intermediate" phase exhibits the same basic monoclinic subcell as $\mathrm{Bi}_{2} \mathrm{TeX}$ (Fig. 2) but a different type of superstructure ordering along the $c$ axis (see more experimental details about the "intermediate" phase in the Supplement, Fig. S8).
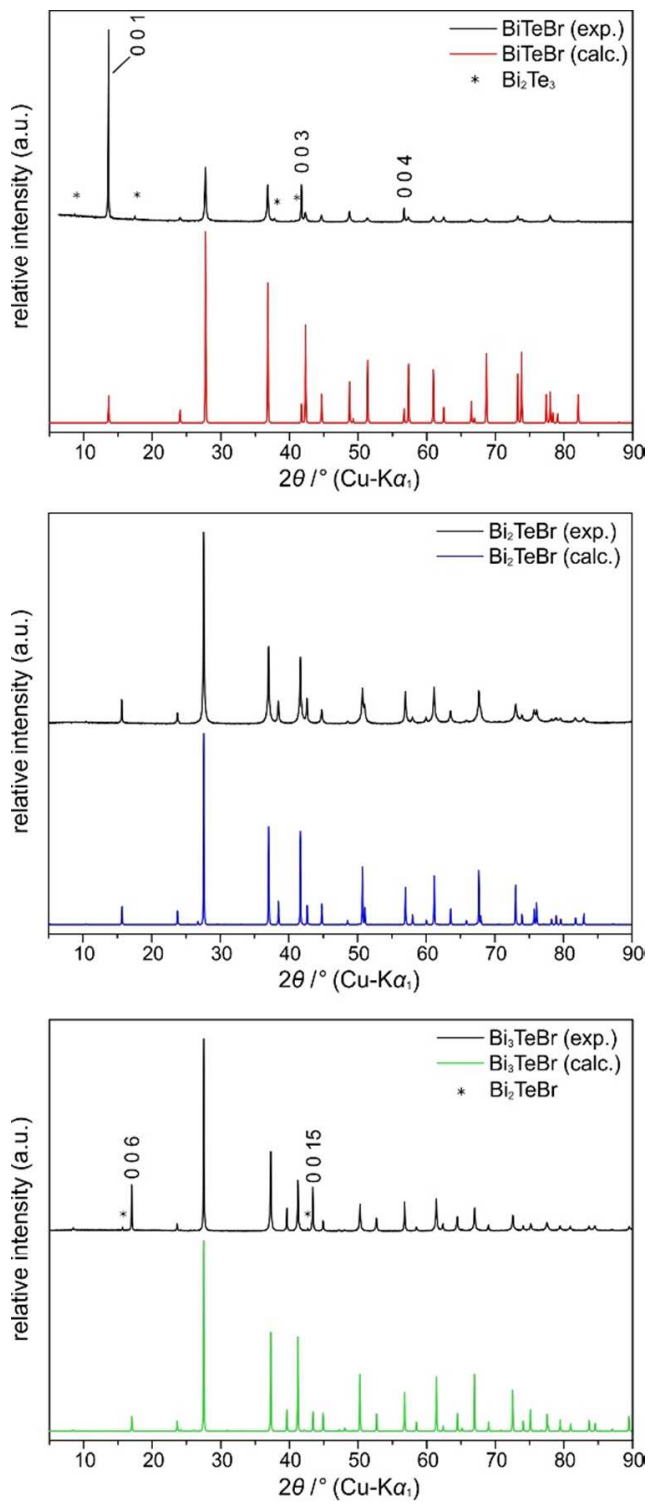

Fig. 1. PXRD patterns of the phase-pure $\mathrm{Bi}_{n} \mathrm{TeBr}$. Results of indexing: $\mathrm{BiTeBr}$ (sp. gr. P3m1) $a=426.88(3)$ pm, $c=648.86(2) \mathrm{pm} ; \mathrm{Bi}_{2} \mathrm{TeBr}$ (sp. gr. $\left.R \overline{3} m\right) a=433.01(1) \mathrm{pm}$, $c=5085.2(2) \mathrm{pm} ; \mathrm{Bi}_{3} \mathrm{TeBr}$ (sp. gr. $\left.R_{3} m\right) a=437.39(1) \mathrm{pm}$, $c=3128.1(1) \mathrm{pm}$.

Table 1: The unit cell parameters (X-ray powder data) and volumes of the ternary compounds in the Bi-Te$X(X=I, B r)$ systems. The PXRD patterns of $B_{n} T e B r$ were indexed by analogy with the respective $B i_{n} T e I$. No superstructure reflections were observed.

\begin{tabular}{cll}
\hline $\boldsymbol{n}$ & $\mathbf{B i}_{\boldsymbol{n}} \mathbf{T e B r}$ & \multicolumn{1}{c}{$\mathbf{B i}_{\boldsymbol{n}} \mathbf{T e I}^{32,33,59}$} \\
\hline $\mathbf{1}$ & $a=427 \mathrm{pm}, c=649 \mathrm{pm}$ & $a=434 \mathrm{pm}, c=685 \mathrm{pm}$ \\
& $V=102.5 \times 10^{6} \mathrm{pm}^{3}$ & $V=111.7 \times 10^{6} \mathrm{pm}^{3}$ \\
$\mathbf{2}$ & $a=433 \mathrm{pm}, c=5085 \mathrm{pm}$ & $a=438 \mathrm{pm}, c=5284 \mathrm{pm}^{*}$ \\
& $V=825.7 \times 10^{6} \mathrm{pm}^{3}$ & $V=877.9 \times 10^{6} \mathrm{pm}^{3}$ \\
3 & $a=437 \mathrm{pm}, c=3128 \mathrm{pm}$ & $a=440 \mathrm{pm}, c=3223 \mathrm{pm}$ \\
& $V=517.3 \times 10^{6} \mathrm{pm}^{3}$ & $V=540.4 \times 10^{6} \mathrm{pm}^{3}$ \\
\hline
\end{tabular}

${ }^{*}$ rhombohedral setting 

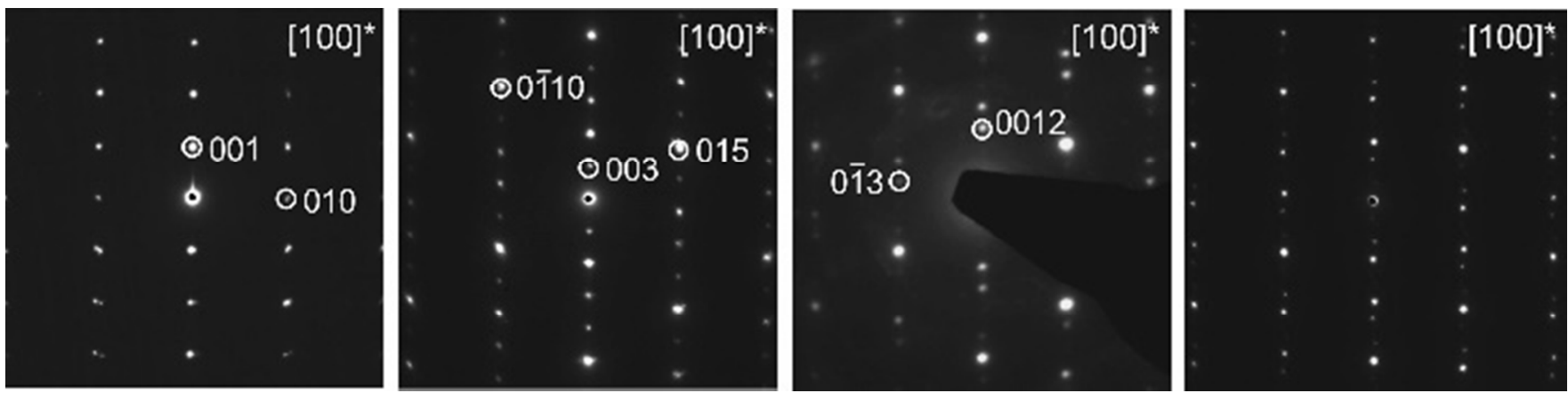

Fig. 2. The main zone $[100]^{*} \mathrm{ED}$ patterns of $\mathrm{BiTeBr}, \mathrm{Bi}_{3} \mathrm{TeBr}, \mathrm{Bi}_{2} \mathrm{TeBr}$ and the "intermediate" phase (left to right). Indexing accords with Table 1.

\section{Crystal Structure Elucidation of $\operatorname{Bi}_{n} \operatorname{TeBr}(n=1,2,3)$}

2.1. Crystal Growth Experiments. As shown above, crystallization from melts is not applicable to $\mathrm{Bi}_{2} \mathrm{TeBr}$ and $\mathrm{Bi}_{3} \mathrm{TeBr}$; hence chemical-vapor-transport (CVT), autotransport or mineralization could be the methods of choice to produce high-quality single crystals.

CVT methods are well-established for BiTeBr. Relying on the known growth mechanism, we obtained BiTeBr crystals of ca. $2 \mathrm{~mm}$ in diameter via autotransport at $450^{\circ} \mathrm{C}$ (plus a small temperature gradient due to internal convection in the tube furnace) for at least 10 days.

Single crystals of $\mathrm{Bi}_{2} \mathrm{TeBr}$ and $\mathrm{Bi}_{3} \mathrm{TeBr}$ (Fig. 3) suitable for X-ray diffraction studies (SCXRD) have grown, presumably via mineralization, directly on the phase-pure powder batches treated at $400^{\circ} \mathrm{C}$ and $385^{\circ} \mathrm{C}$, respectively (see previous section). It was noticed that a longer annealing time favors the formation of larger crystals.

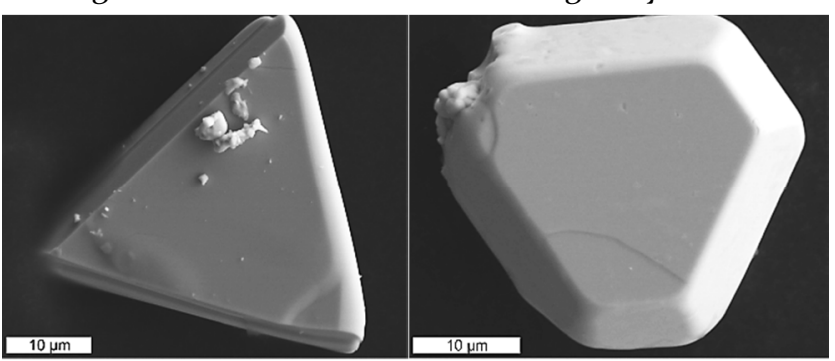

Fig 3. Single crystals of $\mathrm{Bi}_{2} \mathrm{TeBr}$ (left) and $\mathrm{Bi}_{3} \mathrm{TeBr}$ (right) used for SCXRD experiments. EDX data (averaged over 5 crystals (ca. 10 points per each), in at. \%): $\mathrm{Bi}$, 50.7(2); Te, 25.5(2); $\mathrm{Br}, 23.8(1)$ for $\mathrm{Bi}_{2} \mathrm{TeBr}$; $\mathrm{Bi}$, 6o.3(6); Te, 19.3(6); $\mathrm{Br}, 20(1)$ for $\mathrm{Bi}_{3} \mathrm{TeBr}$.

2.2. Crystal Structure of BiTeBr. The first published Rietveld refinement characterized $\mathrm{BiTeBr}$ as a $2 \mathrm{H}-\mathrm{CdI}_{2}-$ type structure with mixed, statistical $\mathrm{Te} / \mathrm{Br}$ occupancy of the anion positions (sp. gr. $P \overline{3} m 1$ ), while BiTeI was found to crystallize in the polar space group (sp. gr. $P_{3} m_{1}$ ) with separated I and Te sites. ${ }^{59}$ Addressing the $\mathrm{Te} / \mathrm{Br}$ disorder issue, relativistic $a b$ initio calculations predict the ordered $\mathrm{BiTeBr}$ to be by $180 \mathrm{meV} / \mathrm{f}$. u. more energetically favorable. ${ }^{60}$ Some recent contributions claim to have confirmed the non-centrosymmetric structure of BiTeBr experimentally by X-ray diffraction, but, as a matter of fact, either no experimental evidence was given, ${ }^{61}$ or a mere Le Bail fit of the unit cell parameters was presented ${ }^{19}$. The absence of an inversion center within the ${ }_{\infty}^{2}[\mathrm{BiTeBr}]$ layers was deduced indirectly from group theory analysis and Raman scattering measurements. ${ }^{19,62}$

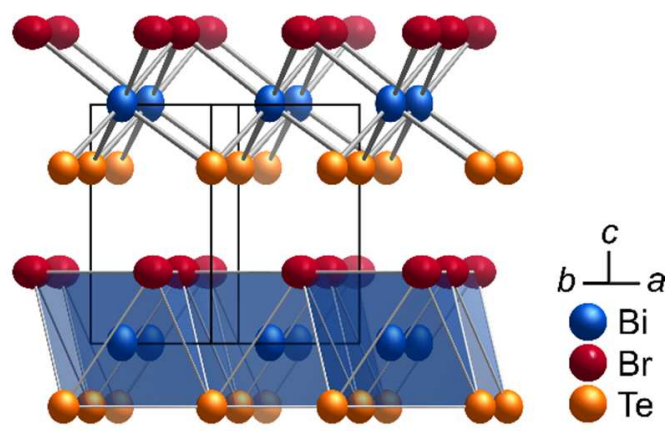

Fig. 4. Ordered BiTeBr crystal structure. Ellipsoids comprise $99 \%$ of the probability density of the atoms.

Our re-determination of the structure by X-ray diffraction on a single-crystal definitely proves that BiTeBr crystallizes in the polar spacegroup $P_{3 m 1}$ (no. 156) and is isostructural to BiTeI. The lattice parameters at room temperature are $a=426.77(1) \mathrm{pm}$ and $c=648.43(3) \mathrm{pm}$ (Table $\mathrm{S}_{1}$ ) in good agreement with the previous data ${ }^{59}$ $(a=426.62(1) \mathrm{pm}$ and $c=648.7(1) \mathrm{pm})$. The trigonal structure of $\mathrm{BiTeBr}$ is closely related to the $\mathrm{CdI}_{2}$ structure type and is assembled by ${ }_{\infty}^{2}\left[\mathrm{BiTe}_{3 / 3} \mathrm{Br}_{3 / 3}\right]$ triple layers (Fig. 4). In contrast to the hitherto disputed mixed occupancy of $\mathrm{Te}$ and $\mathrm{Br},{ }^{59}$ our refinement yields anionic ordering in two separate atomic positions (Table S2) in full accordance with [61]. Given the non-centrosymmetric structures of both $\mathrm{BiTeBr}$ and $\mathrm{BiTeI}$, statistical $\mathrm{Br} / \mathrm{I}$ mixing can be assumed in the $\mathrm{BiTeI}_{1-\mathrm{x}} \mathrm{Br}_{\mathrm{x}}$ series. Halogen ordering would result in the decreased symmetry and a different set of the unique reflections in PXRD.

2.3. Crystal Structures of $\mathrm{Bi}_{2} \mathrm{TeBr}$ and $\mathrm{Bi}_{2} \mathrm{TeI}$. Our $\mathrm{X}$ ray diffraction experiment on a $\mathrm{Bi}_{2} \mathrm{TeBr}$ single crystal reveals a rhombohedral lattice with $a=433.04(2) \mathrm{pm}$ and $c=5081.6(3) \mathrm{pm}$ and the centrosymmetric space group $R \overline{3} m$ (no. 166) (Table 2). The crystal structure can be described, just like $\mathrm{Bi}_{2} \mathrm{TeI}^{32,33}$, as a regular alternation of one buckled ${ }_{\infty}^{2}\left[\mathrm{Bi}_{2}\right]$ layer and two consecutive ${ }_{\infty}^{2}\left[\mathrm{BiTe}_{3 / 3} \mathrm{Br}_{3 / 3}\right]$ trilayers (Fig. 5, Table $\mathrm{S}_{4}$ ). 


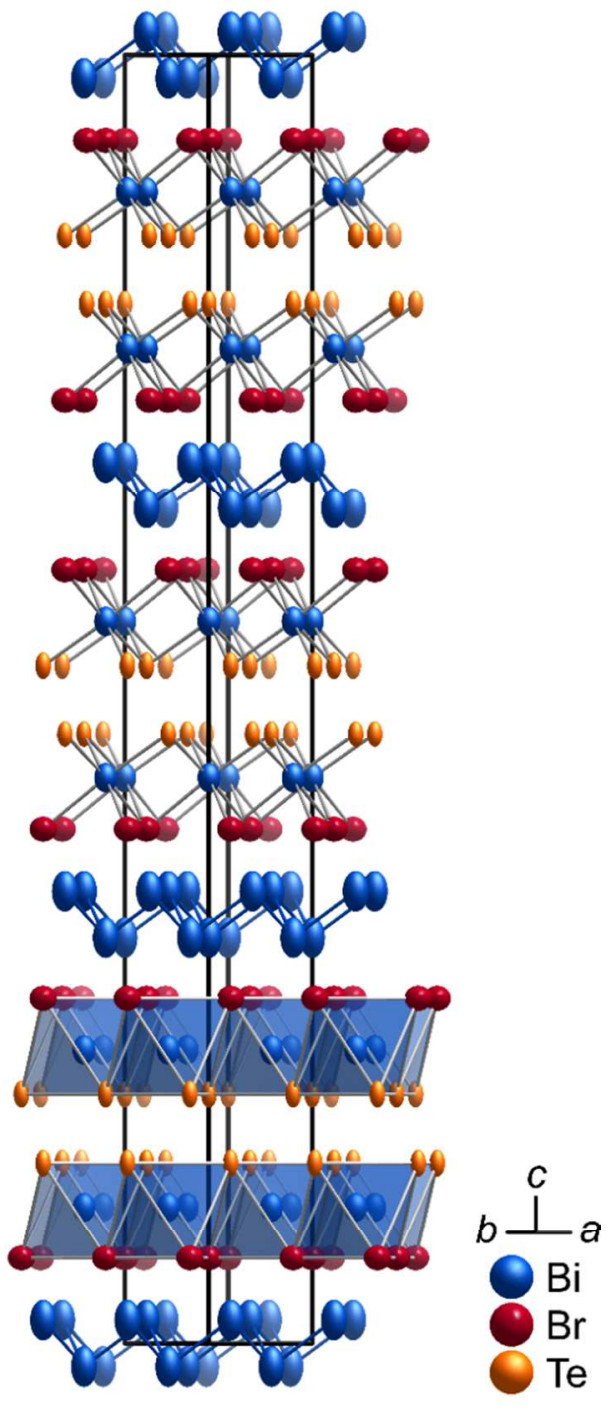

Fig. 5. $\mathrm{Bi}_{2} \mathrm{TeBr}$ crystal structure. Ellipsoids comprise $99 \%$ of the probability density of the atoms.

The crystal structure of $\mathrm{Bi}_{2} \mathrm{TeI}$ was initially solved in the C-centered monoclinic cell. ${ }^{32}$ We showed ${ }^{33}$ a possible transformation into a cell with trigonal (rhombohedral) metrics. This transformation causes negligible deviations

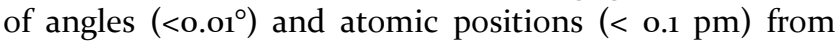
the higher symmetric space group $R \overline{3} \mathrm{~m}^{33}$. Overlooked trigonal symmetry is found relatively often in the previously reported $C$-centered monoclinic structures. ${ }^{63}$ We hereby revisit the single-crystal solution for $\mathrm{Bi}_{2} \mathrm{TeI}$ and describe it in the centrosymmetric space group $R \overline{3} m$ (no. 166) with $a=437.88(1) \mathrm{pm}$ and $c=5283.8(3) \mathrm{pm}$ (Table 2 , Table S6).

The final $R$ values and the residual electron density indicate crystallographic problems in both isostructural
$\mathrm{Bi}_{2} \mathrm{Te} X$, likely stemming from structural disorder. It may be caused by the presence of an inversion center in the $\mathrm{Bi}_{2} \mathrm{Te} X$ structures, which is generated via the flipping of every second ${ }_{\infty}^{2}\left[\mathrm{BiTe}_{3 / 3} X_{3 / 3}\right]$ trilayer, in contrast to the polar stacking of $\mathrm{BiTe} X$. Calculations demonstrate that both mutual orientations of the trilayers, [I-Bi-Te-Te-BiI] and [I-Bi-Te-I-Bi-Te] differ negligibly by $0.5 \mathrm{meV}^{64}$, making stacking disorder in bulk highly probable. On the other hand, the calculated formation energy of stacking faults in bulk $\mathrm{BiTeBr}$ is about 50 times higher than in BiTeI. ${ }^{65}$

The shapes and sizes of the displacement ellipsoids (Table S4, Table S6) suggest a certain type of stacking disorder in $\mathrm{Bi}_{2} \mathrm{Te} X$ that is associated with varying stacking sequences of modules with different chemical identity rather than shifts of modules. Our TEM study do not, however, lend a corroboration for this assumption.

2.4. Crystal Structure of $\mathbf{B i}_{3} \mathbf{T e B r}$. The most Bi-rich compound of the presented series, $\mathrm{Bi}_{3} \mathrm{TeBr}$, crystallizes in the polar space group $R_{3} m$ (no. 16o) with lattice parameters $a=437.68(3) \mathrm{pm}$ and $c=3122.9(3) \mathrm{pm}$ (Table 3 ) and is isostructural to $\mathrm{Bi}_{3} \mathrm{TeI}^{33}$. It is built up by alternating corrugated ${ }_{\infty}^{2}\left[\mathrm{Bi}_{2}\right]$ bilayers and ${ }_{\infty}^{2}\left[\mathrm{BiTe}_{3 / 3} \mathrm{Br}_{3 / 3}\right]$ trilayers (Fig. 6 , Table S8). $\mathrm{Bi}_{3}$ TeI was described with the aid of a pseudoinversion center in order to explain the conspicuous displacement parameters. ${ }^{33}$ Thanks to larger domains in $\mathrm{Bi}_{3} \mathrm{TeBr}$ promoting higher resolution, it is now possible to improve this description to some extent. The crystal structure of $\mathrm{Bi}_{3} \mathrm{TeBr}$ was refined with reverse-obverse (a result of two settings in the rhombohedral cell) and inversion twinning. Additionally, a split position was introduced on both the Te1 and the Biz site, and the respective occupancies of the split atoms were refined to $40 \%$ of $\mathrm{Bi}$ in the Ter site, and $40 \%$ of Te in the Biz site, respectively (Fig. S1o). Regardless of the applied description, this recurring crystallographic problem is most likely caused by ubiquitous stacking disorder, originating from the small energy differences between various stacking variants of the ${ }_{\infty}^{2}\left[\mathrm{BiTe}_{3 / 3} X_{3 / 3}\right]$ trilayers with respect to each other and to the ${ }_{\infty}^{2}\left[\mathrm{Bi}_{2}\right]$ layers. The sizes of the thermal displacement parameters for $\mathrm{Te}$ and $\mathrm{Br}$ compared between $\mathrm{BiTeBr}(\mathrm{Ta}-$ ble $\mathrm{S}_{2}$ ) and $\mathrm{Bi}_{3} \mathrm{TeBr}$ (Table S8) may point at such disorder type as flipping of ${ }_{\infty}^{2}\left[\mathrm{BiTe}_{3 / 3} \mathrm{Br}_{3 / 3}\right]$ trilayers.

We have also considered yet another possible stacking sequence for $\mathrm{Bi}_{3} \mathrm{TeBr}$ with two ${ }_{\infty}^{2}\left[\mathrm{BiTe}_{3 / 3} \mathrm{Br}_{3 / 3}\right]$ trilayers followed by two ${ }_{\infty}^{2}\left[\mathrm{Bi}_{2}\right]$ bilayers that face only the $\mathrm{Br}$ atomic planes along the $c$ axis. This model bears more similarity to the $\mathrm{Bi}_{2} \mathrm{Te} X$ stacking since the interlayer $\mathrm{Bi}-\mathrm{Te}$ contacts would be also avoided. This structure hypothesis complies with the X-ray diffraction data, but is not supported by the HRTEM imaging (cf. Fig $\mathrm{S}_{7}$ ). 
Table 2: Crystallographic data for $\mathrm{Bi}_{2} \mathrm{TeBr}$ and $\mathrm{Bi}_{2} \mathrm{TeI}$ (refined in a rhombohedral cell).

\begin{tabular}{|c|c|c|}
\hline Compound & $\mathrm{Bi}_{2} \mathrm{TeBr}$ & $\mathrm{Bi}_{2} \mathrm{TeI}$ \\
\hline Crystal system, space group & \multicolumn{2}{|c|}{ Trigonal, $R \overline{3} m$ (no. 166) } \\
\hline Formula units & \multicolumn{2}{|c|}{$Z=6$} \\
\hline Lattice parameters & $\begin{array}{l}a=433.04(2) \mathrm{pm} \\
c=5081.6(3) \mathrm{pm} \\
V=825.3(1) \times 10^{6} \mathrm{pm}^{3} \\
\rho_{\text {calc. }}=7.55 \mathrm{~g} \mathrm{~cm}^{-3}\end{array}$ & $\begin{array}{l}a=437.88(1) \mathrm{pm} \\
c=5283.8(2) \mathrm{pm} \\
V=877.4(1) \times 10^{6} \mathrm{pm}^{3} \\
\rho_{\text {calc. }}=7.64 \mathrm{~g} \mathrm{~cm}^{-3}\end{array}$ \\
\hline Range for data collection; index ranges & $\begin{array}{l}4.81^{\circ} \leq 2 \theta \leq 49.71^{\circ}(\lambda=71.073 \mathrm{pm}) \\
-5 \leq h \leq 5,-5 \leq k \leq 4,-60 \leq l \leq 54\end{array}$ & $\begin{array}{l}6.94^{\circ} \leq 2 \theta \leq 49.99^{\circ}(\lambda=71.073 \mathrm{pm}) \\
-5 \leq h \leq 5,-5 \leq k \leq 5,-62 \leq l \leq 62\end{array}$ \\
\hline Collected reflections & 3230 measured, 230 unique & 3742 measured, 403 unique \\
\hline $\mathrm{R}$ indices of merging & $R_{\mathrm{int}}=0.033, R_{\sigma}=0.013$ & $R_{\mathrm{int}}=0.030, R_{\sigma}=0.015$ \\
\hline Structure refinement & \multicolumn{2}{|c|}{$\begin{array}{l}\text { Full-matrix least-squares based on } F^{2} \text {, } \\
\text { anisotropic displacement parameters; }\end{array}$} \\
\hline Data/restraints/parameters & $230 / 0 / 13$ & $403 / 0 / 14$ \\
\hline Final $\mathrm{R}$ indices and goodness-of-fit on $\mathrm{F}^{2}$ & $\begin{array}{l}R_{1}\left[181 F_{\mathrm{o}}>4 \sigma\left(F_{\mathrm{o}}\right)\right]=0.036 \\
w R_{2}\left(\text { all } F_{\mathrm{o}}^{2}\right)=0.065 \\
\text { Goo } F=1.223\end{array}$ & $\begin{array}{l}R_{1}\left[181 F_{\mathrm{o}}>4 \sigma\left(F_{\mathrm{o}}\right)\right]=0.042 \\
w R_{2}\left(\text { all } F_{\mathrm{o}}^{2}\right)=0.092 \\
\text { GooF }=1.101\end{array}$ \\
\hline Min./max. residual electron density & $-3.22 / 2.05 \mathrm{e} \times 10^{-6} \mathrm{pm}^{-3}$ & $-5.26 / 4.05 \mathrm{e} \times 10^{-6} \mathrm{pm}^{-3}$ \\
\hline
\end{tabular}




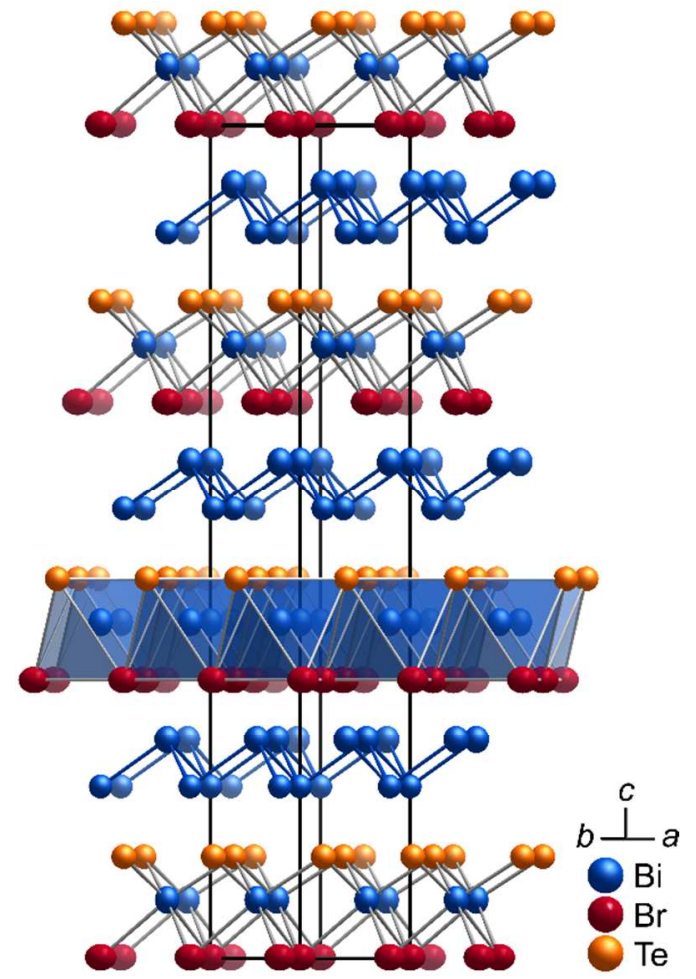

Fig. 6. $\mathrm{Bi}_{3} \mathrm{TeBr}$ crystal structure. Ellipsoids comprise $99 \%$ of the probability density of the atoms.

Table 3: Crystallographic data for $\mathrm{Bi}_{3} \mathrm{TeBr}$.

\begin{tabular}{|c|c|}
\hline Crystal system, space group & Trigonal, $R_{3} m$ (no. 16o) \\
\hline Formula units & $Z=3$ \\
\hline Lattice parameters & $\begin{array}{l}a=437.68(3) \mathrm{pm} \\
c=3122.9(3) \mathrm{pm} \\
V=518.1(1) \times 10^{6} \mathrm{pm}^{3} \\
\rho_{\text {calc. }}=8.03 \mathrm{~g} \mathrm{~cm}^{-3}\end{array}$ \\
\hline $\begin{array}{l}\text { Range for data collection; } \\
\text { index ranges }\end{array}$ & $\begin{array}{l}3.92^{\circ} \leq 2 \theta \leq 72.62^{\circ} \\
(\lambda=71.073 \mathrm{pm}) ; \\
-7 \leq h \leq 7,-7 \leq k \leq 7,-51 \leq l \\
\leq 49 ;\end{array}$ \\
\hline Collected reflections & 8650 measured, 1193 unique \\
\hline$R$ indices of merging & $R_{\mathrm{int}}=0.051, R_{\sigma}=0.033$ \\
\hline Structure refinement & $\begin{array}{l}\text { Full-matrix least-squares } \\
\text { based on } F^{2} \text {, anisotropic } \\
\text { displacement parameters; }\end{array}$ \\
\hline Data/restraints/parameters & $1193 / 0 / 22$ \\
\hline $\begin{array}{l}\text { Final } R \text { indices and good- } \\
\text { ness-of-fit on } F^{2}\end{array}$ & $\begin{array}{l}R_{1}\left[289 F_{\mathrm{o}}>4 \sigma\left(F_{\mathrm{o}}\right)\right]=0.022 \\
w R_{2}\left(\text { all } F_{\mathrm{o}}^{2}\right)=0.045 \\
\text { Goo } F=1.005\end{array}$ \\
\hline $\begin{array}{l}\text { Min./max. residual elec- } \\
\text { tron density }\end{array}$ & $-1.02 / 0.60 \mathrm{e} \times 10^{-6} \mathrm{pm}^{-3}$ \\
\hline
\end{tabular}

An in-depth theoretical and spectroscopic study of structural disorder in $\mathrm{Bi}_{n} \mathrm{Te} X$ could be highly relevant for their physical properties. Theory has found a connection between such defects as flipped trilayers and topological properties. ${ }^{64,66}$ For instance, two adjacent ${ }_{\infty}^{2}[\mathrm{BiTeI}]$ trilayers connected via an inversion center and facing their Te atomic planes across the van der Waals gap would form a $2 \mathrm{D}$ topological defect in the trivial noncentrosymmetric BiTeI bulk. Moreover, a hypothetical periodic lattice built by regularly stacked $[\mathrm{Br}-\mathrm{Bi}-\mathrm{Te} \cdots \mathrm{Te}-$ $\mathrm{Bi}-\mathrm{Br}$ ] fragments could be a strong ${ }_{3} \mathrm{D}$ TI. It is, however, notably less favorable (by ca. $20 \mathrm{meV} / \mathrm{f}$. u.) than the noncentrosymmetric bulk BiTeBr. This prediction was confirmed by an STM study of BiTeBr ${ }^{65}$ revealing two singledomain (oo1) surfaces with opposite terminations. On the contrary, BiTeI has a multi-domain (ool) surface with coexisting Te- and I-terminations on the scale of $100 \mathrm{~nm} .{ }^{11,67^{-}}$ ${ }^{69}$ Consequently, $\mathrm{BiTeBr}$ is believed to be the most homogeneous of all $\mathrm{BiTe} X$ tellurohalides and a well-suited candidate for future spintronic applications. We also did not observe any signs of stacking disorder, domains or flipped layers in BiTeBr. As opposed to that, both Bi-rich tellurobromides and telluroiodides are prone to stacking disorder. Nevertheless, new $\mathrm{Bi}_{n} \mathrm{TeBr}$ may become a better playground for forthcoming investigations, because twinning phenomena can be resolved better than in $\mathrm{Bi}_{n} \mathrm{TeI}$.

Comparison of the interatomic distances in $\mathrm{Bi}_{n} \mathrm{Te} X(X=$ $\mathrm{I}, \mathrm{Br})$ hints at similar bonding scenarios in the respective compounds (Table 4, Table $S_{3}$, Table $S_{5}$, Table $S_{7}$, Table $\mathrm{S} 9$ ). The $\mathrm{Bi}-\mathrm{Te}$ distances are virtually unchanged as one moves from a telluroiodide to the corresponding tellurobromide. An exception observed for $\mathrm{Bi}_{3} \mathrm{TeBr}$ is most likely a consequence of the refinement of a split position that entails a small shift of the Te atom towards the Bi atom. Since $\mathrm{Bi}_{3} \mathrm{TeI}$ was refined without a split position, the estimated interatomic distance is slightly longer. As can be expected from the electronegativity values, the $\mathrm{Bi}-X$ distances are significantly shorter for $X=\mathrm{Br}$ than for $X=\mathrm{I}$. A general tendency towards elongation of pairwise bonds and diminishing van der Waals gaps upon increasing $n$ is observed for all compounds. Interlayer and intralayer $\mathrm{Bi}-$ $X$ contacts become almost equal in $\mathrm{Bi}_{3} \mathrm{TeBr}$ as an effect of the introduced inversion twin $(50 / 50)$, but also due to strengthening metallicity and interlayer interactions within the stack (cf. next section). A larger portion of Bibilayers enhances the metallic character of the discussed compounds.

We analyzed changes in the unit cell volumes upon bromine substituting for iodine in the entire $\mathrm{Bi}_{n} \mathrm{Te} X$ series by comparison with Biltz volume increments ${ }^{55}$. The values for $\mathrm{Br}^{-}\left(25 \mathrm{~cm}^{3} / \mathrm{mol}\right)$ and $\mathrm{I}^{-}\left(34 \mathrm{~cm}^{3} / \mathrm{mol}\right)$ amount to $41.5 \AA^{3}$ and $56.5 \AA^{3}$, respectively. ${ }^{55}$ Thus, a simple size effect by iodine substitution for bromine would yield ca. $36 \%$ increase in volume. However, the experimentally observed volume increments per ${ }_{\infty}^{2}[\mathrm{BiTe} X]$ layer are $9.2 \AA^{3}$ for $n=1$, $8.7 \AA^{3}$ for $n=2$, and only $7.7 \AA^{3}$ for $n=3$, when moving from $\mathrm{Bi}_{n} \mathrm{TeBr}$ to the respective $\mathrm{Bi}_{n}$ TeI. The observed tendencies of decreasing increments upon increasing $n$ could indicate successive strengthening of bonding between the ${ }_{\infty}^{2}[\mathrm{BiTe} X]$ and ${ }_{\infty}^{2}\left[\mathrm{Bi}_{2}\right]$ layers across the van der Waals gap that goes beyond electrostatic anion-anion repulsion and weak van der Waals bonding in pristine 
$\mathrm{BiTe} X$. In other words, differences in electronegativity and polarizability between iodide and bromide become less prominent with increasing $n$ and growing metallicity (see next section).

Table 4: Interatomic distances of $\mathrm{Bi}_{n} \mathrm{Te} X(X=\mathrm{I}, \mathrm{Br}$; $n=1,2,3)$.

\begin{tabular}{llll}
\hline $\boldsymbol{n}$ & Bond & $\mathbf{B i}_{\boldsymbol{n}} \mathbf{T e B r} / \mathbf{p m}$ & $\mathbf{B i}_{\boldsymbol{n}} \mathbf{T e I} / \mathbf{p m}$ \\
\hline $\mathbf{1}$ & $\mathrm{Bi}-\mathrm{Te}$ & $301.1(1)^{\#}$ & $303.9^{+}$ \\
& $\mathrm{Bi}-X$ & $312.1(1)^{\#}$ & $327.3^{+}$ \\
\hline 2 & $\mathrm{Bi}-\mathrm{Te}$ & $304.8(2)^{\#}$ & $305.9(2)^{\#}$ \\
& $\mathrm{Bi}-X$ & $321.6(2)^{\#}$ & $339.1(2)^{\#}$ \\
& $\mathrm{Bi}-\mathrm{Bi}$ & $309.2(2)^{\#}$ & $309.9(2)^{\#}$ \\
3 & $\mathrm{Bi}-\mathrm{Te}$ & $\mathbf{2 9 8 . 8}(5)^{\#}$ & $311.9^{*}$ \\
& $\mathrm{Bi}-X$ & $334.4(2)^{\#}$ & $343.6^{*}$ \\
& $\mathrm{Bi}-\mathrm{Bi}$ & $310.4(4)^{\#}$ & $304.8^{*}$ \\
\hline
\end{tabular}

${ }^{\#}$ : this work, ${ }^{*}$ : [26], ${ }^{+}:[43]$

\section{Electronic Structures of $\operatorname{Bi}_{n} \operatorname{TeBr}(n=2,3)$}

The bulk electronic structure of $\mathrm{Bi}_{2} \mathrm{TeBr}$ with spin-orbit coupling (Fig. 7) has a nearly semimetalic character with electron pockets along the $\Gamma-\mathrm{M}$ and $\mathrm{A}-\mathrm{L}$ directions of the ${ }_{3} \mathrm{D}$ Brillouin zone (BZ). It can be concluded that a single pocket is projected onto the $\bar{\Gamma}-\bar{M}$ direction of the $2 \mathrm{D} \mathrm{BZ}$. A complex inversion involving several groups of bands close to the Fermi level is observed near the $\Gamma$ point. Two band groups reside in the occupied and another two at the unoccupied part of the spectrum. These groups are formed by the states of two different types of building modules: ${ }_{\infty}^{2}[\mathrm{BiTeBr}]$ trilayers (magenta in Fig. 7) and ${ }_{\infty}^{2}\left[\mathrm{Bi}_{2}\right]$ bilayers (blue in Fig. 7). The trilayer states mostly have the $\mathrm{p}_{\mathrm{z}}$ symmetry, whereas the bilayer states possess the $\mathrm{p}_{\mathrm{xy}}$ symmetry. The inversion of these states is caused by a complex hybridization and results in new insulating local band gaps out of the time-reversal invariant momenta (Fig. 7, red dashed ovals). The same inversion scheme has been previously observed for a dual topological insulator $\mathrm{Bi}_{2}$ TeI. ${ }^{43}$ However, the distance between the electron and the hole part of the spectrum is larger in $\mathrm{Bi}_{2} \mathrm{TeI}$, which leads to a semiconductor-like band structure. Such complex inversion of the bulk states in $\mathrm{Bi}_{2} \mathrm{TeBr}$ is a direct indication of the non-trivial topology. Subsequently, topological surface states emerge.

The stacking sequence of the structure modules in $\mathrm{Bi}_{2} \mathrm{TeBr}$ offers three plausible terminations of the natural cleaving, i. e. by $\mathrm{Te}, \mathrm{Br}$ and $\left[\mathrm{Bi}_{2}\right]$-layers.

The electronic structure of the Te-terminated surface is given in Fig. 8a. It features a topological surface state (TSS) in the local bulk band gap close to the Fermi level along the $\bar{\Gamma} \overline{\mathrm{K}}$ and $\bar{\Gamma} \overline{\mathrm{M}}$ directions. The state originates from the complex band inversion and the details of its formation were discussed for the identical Te-termination in $\mathrm{Bi}_{2} \mathrm{TeI}^{43}$. The TSS is gapless along $\bar{\Gamma} \overline{\mathrm{M}}$, whereas there is a tiny gap $(\sim 7 \mathrm{meV})$ in the $\bar{\Gamma} \overline{\mathrm{K}}$ direction. In contrast to the earlier monoclinic description of $\mathrm{Bi}_{2} \mathrm{TeI}^{32,43}$, the trigonal crystal symmetry of $\mathrm{Bi}_{2} \mathrm{Te} X$ necessitates the equivalence of all $\bar{\Gamma} \bar{M}$ directions and the gapless degeneracy of TSS along them.

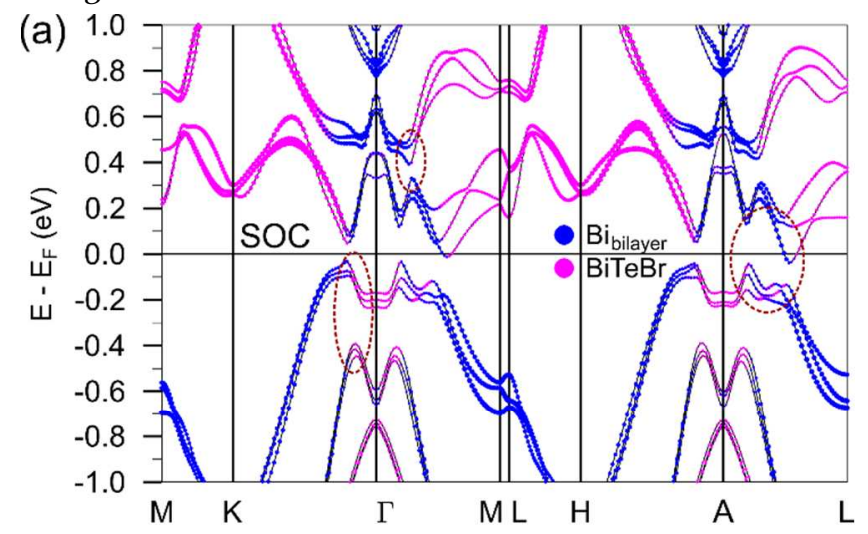

(b)

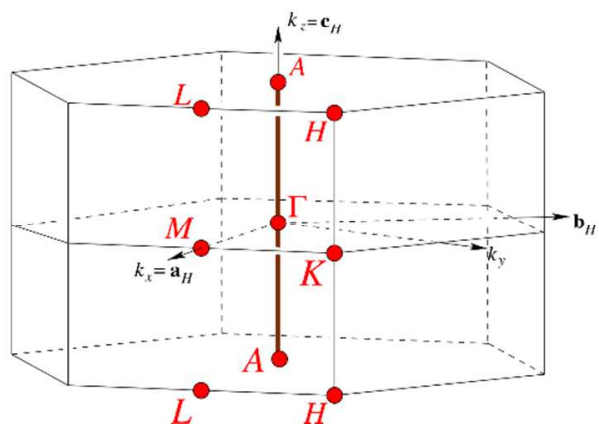

Fig. 7. a) Bulk electronic structure of $\mathrm{Bi}_{2} \mathrm{TeBr}$ with spin-orbit coupling. The states of the ${ }_{\infty}^{2}[\mathrm{Te}-\mathrm{Bi}-\mathrm{Br}]$ trilayers $\left(\underset{\infty}{2}\left[\mathrm{Bi}_{2}\right]\right.$-layers) are marked in magenta (blue). The local gap formed via a complex hybridization is outlined by dashed red ovals. b) The corresponding $3 \mathrm{D} \mathrm{BZ}$.
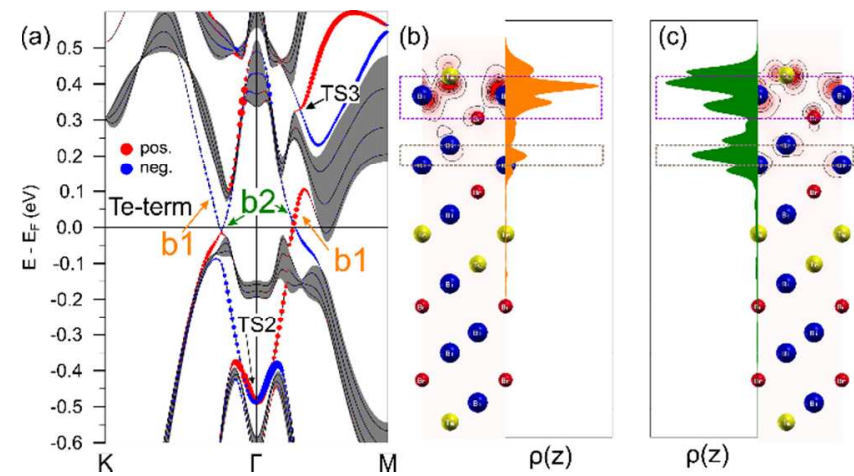

Fig. 8. (a) Surface electronic structure of $\mathrm{Bi}_{2} \mathrm{TeBr}$ for the Te-termination. The bulk band continuum is shown in grey. For surface states, the radii of the red and blue circles symbolize the values of the in-plane components of spin eigenvalues perpendicular to the shown direction of the $2 \mathrm{D}$ Brillouin zone. (b) Spatial distribution of the charge density for the bi branch in the (1120) plane near the cross point (left panel). The maximum and minimum charge-density value of the state are marked by red and white, respectively. The charge density $\rho(z)$ of the branch integrated over the $x y$ plane is shown in orange (right panel). Lila 
and grey dotted rectangles outline the topmost surficial trilayer and bilayer modules. (c) Ibid, for the b2 branch.

The TSS has two branches (marked b1 and b2 in Fig. 8a) with different spatial localization and symmetry of the atomic orbitals. The outer branch (marked by b1) is strongly localized in the topmost ${ }_{\infty}^{2}[\mathrm{Te}-\mathrm{Bi}-\mathrm{Br}]$ layer and is mostly formed by the $\mathrm{p}_{\mathrm{z}}$ orbitals of the topmost Te atoms and the $\mathrm{Bi}$ p-orbitals of this trilayer (see Fig. 8b). The more remote $\mathrm{Br}$ atoms and ${ }_{\infty}^{2}\left[\mathrm{Bi}_{2}\right]$-layers make only a minor contribution to b1. The inner branch b2 is localized in both ${ }_{\infty}^{2}[\mathrm{Te}-\mathrm{Bi}-\mathrm{Br}]$ and adjacent ${ }_{\infty}^{2}\left[\mathrm{Bi}_{2}\right]$ modules (Fig. 8bc) and is composed of the p-orbitals of both the topmost trilayer and bilayer modules. In contrast to bi, the contributing orbital symmetry in the topmost ${ }_{\infty}^{2}[\mathrm{Te}-\mathrm{Bi}-\mathrm{Br}]$ trilayer and ${ }_{\infty}^{2}\left[\mathrm{Bi}_{2}\right]$-bilayer changes: the Te-states have the $\mathrm{p}_{\mathrm{xy}}$ symmetry, the $\mathrm{Br}$-states have the $\mathrm{p}_{\mathrm{z}}$ symmetry, and the $\left[\mathrm{Bi}_{2}\right]$-states have the $\mathrm{p}_{\mathrm{xy}}$ symmetry.

The complex inversion of the bulk states also fosters additional spin-polarized TSS with the Dirac-like dispersion in the valence and conduction bands (denoted TS2 and $\mathrm{TS}_{3}$ in Fig. 8a).

The surface electronic structure of the $\mathrm{Br}$-terminated surface of $\mathrm{Bi}_{2} \mathrm{TeBr}$ in shown in Fig. 9. It is characterized by both trivial and topological surface states. The former (SS1) sits in the local bulk band gap near the Fermi level and stems from an effective electric field gradient that is normal to the surface plane. The gradient occurs in the topmost asymmetric trilayer, and, consequently, the $\mathrm{SS}_{1}$ is predominantly localized in there (lila profile in Fig. 9b). In agreement with the electric field gradient, the spinsplitting and the dispersion of SS1 have a Rashba-like character. The TSS resides in both electron and hole local band gaps. In contrast to the Te-termination, the state is present in the local gap near the Fermi level only along the $\bar{\Gamma} \bar{M}$ directions of the $2 \mathrm{D}$ BZ. The spatial distribution profiles for both branches (b1 and b2) resemble those at the Te-terminated surface, but the state at the $\mathrm{Br}$-surface penetrates much deeper into the $[\mathrm{Br}-\mathrm{Bi}-\mathrm{Te}]$ topmost trilayer. Actually, a gapless spin-helical TSS is found only on the Te-terminated surface. On the Br-surface, it survives only along the $\bar{\Gamma} \overline{\mathrm{M}}$ direction due to hybridization with the trivial Rashba-type surface state.

An additional spin-polarized TSS (TS2, Fig. 9) exists in the local valence band gap. It is also caused by the complex inversion involving several bulk bands. In contrast to the TSS close to the Fermi level, the state in the electron part of the spectrum has a linear dispersion and forms a Dirac cone with branches uniformly localized within the outer trilayer fragments (Fig. 9b).

The bulk electronic structure of $\mathrm{Bi}_{3} \mathrm{TeBr}$ without spinorbit coupling is similar to that for $\mathrm{Bi}_{3} \mathrm{Tel}^{33}$ (Fig. S12). When spin-orbit coupling is taken into account, the semimetallic electronic spectrum of $\mathrm{Bi}_{3} \mathrm{TeBr}$ transforms into a metallic one (Fig. 10). Interactions between the two structure fragments, ${ }_{\infty}^{2}\left[\mathrm{Bi}_{2}\right]$ and ${ }_{\infty}^{2}[\mathrm{BiTeBr}]$, trigger a complex hybridization of the bulk states. In particular, this hybridization produces several local gaps with inverted edges (marked by green ovals in Fig. 10). Like $\mathrm{Bi}_{2} \mathrm{TeBr}$ and $\mathrm{Bi}_{2} \mathrm{TeI}^{33}$ and in contrast to isostructural $\mathrm{Bi}_{3} \mathrm{TeI}$, the inversion in $\mathrm{Bi}_{3} \mathrm{TeBr}$ occurs between the states of two distinct structure fragments, corroborating the modular principle. The inversion close to the Fermi level proceeds predominately between the $\mathrm{p}_{\mathrm{x}}$ - and the $\mathrm{p}_{\mathrm{y}}$-states of the ${ }_{\infty}^{2}[\mathrm{BiTeBr}]$ trilayer and the $\mathrm{p}_{\mathrm{z}}$-states of ${ }_{\infty}^{2}\left[\mathrm{Bi}_{2}\right]$ (Fig. 10).

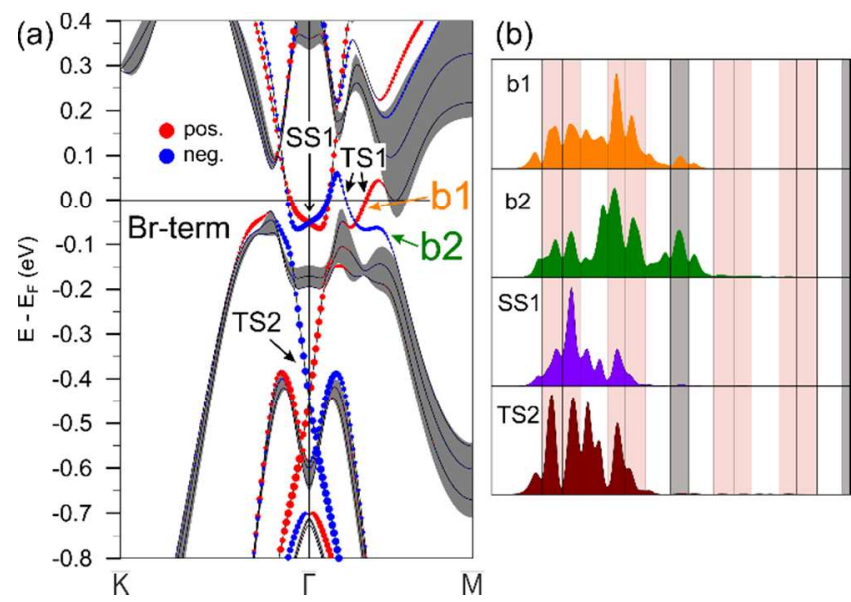

Fig. 9. (a) Surface electronic structure of $\mathrm{Bi}_{2} \mathrm{TeBr}$ for the Br-termination. The bulk band continuum is shown in grey. For surface states, the radii of the red and blue circles symbolize the values of the in-plane components of spin eigenvalues perpendicular to the shown direction of the $2 \mathrm{D}$ Brillouin zone. (b) Charge density of states integrated over the $x y$ plane for the points shown by arrows in the panel (a).

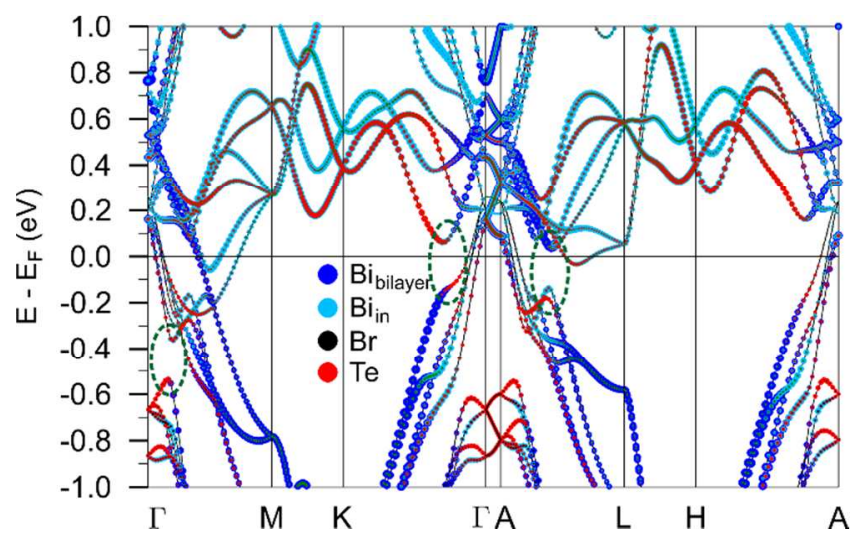

Fig. 10. Bulk electronic structure of $\mathrm{Bi}_{3} \mathrm{TeBr}$ with spin-orbit coupling with atomic orbital contributions. The dark green ovals encircle local gaps formed via complex hybridization.

Fig. 11 and Fig. 12 present the surface electronic structure of $\mathrm{Bi}_{3} \mathrm{TeBr}$ with spin texture for all four possible surface terminations. The terminations of all types host spinpolarized TSS that stem from the above discussed bulkband inversion. A topological surface state on the Teterminated surface represents the upper part of a Dirac cone and is formed by two branches propagating in the $\bar{\Gamma} \overline{\mathrm{K}}$ and $\bar{\Gamma} \overline{\mathrm{M}}$ directions (Fig. 11a). The Dirac point is submerged into the bulk continuum of the valence band. The 
surface states on the Br-terminated (Fig. 11b) and two types of the Bi-terminated (Fig. 12) surfaces are localized over a wide energy range (from -0.6 to o eV) in the local valence band gap. These states have a cone-like dispersion with the Dirac point at the center of the Brillouin zone. The energy position of the cone is directly determined by the near-surface band-bending effect, which can be traced by a comparison of the DFT and the tightbinding results (right panels in Fig. 11, 12). The latter data do not comprise the band-bending contribution. $\mathrm{The} \mathrm{Br}-$ terminated surface exhibits positive band-bending, while the remaining $\mathrm{Te}$ - and Bi2-terminations demonstrate a negative effect. Due to particularly large negative band bending at the Te-terminated surface, the cone dispersion is distorted by interaction with the bulk-band continuum at approx. $-0.6 \mathrm{eV}$. Hence the Te-terminated surface is characterized by maximal negative band-bending, whereas the type- ${ }_{\infty}^{2}\left[\mathrm{Bi}_{2}\right]$-termination exhibits the largest positive band-bending. Note that the band-bending effect is larger on the type-II ${ }_{\infty}^{2}\left[\mathrm{Bi}_{2}\right]$-terminated surface than on the type-I one.
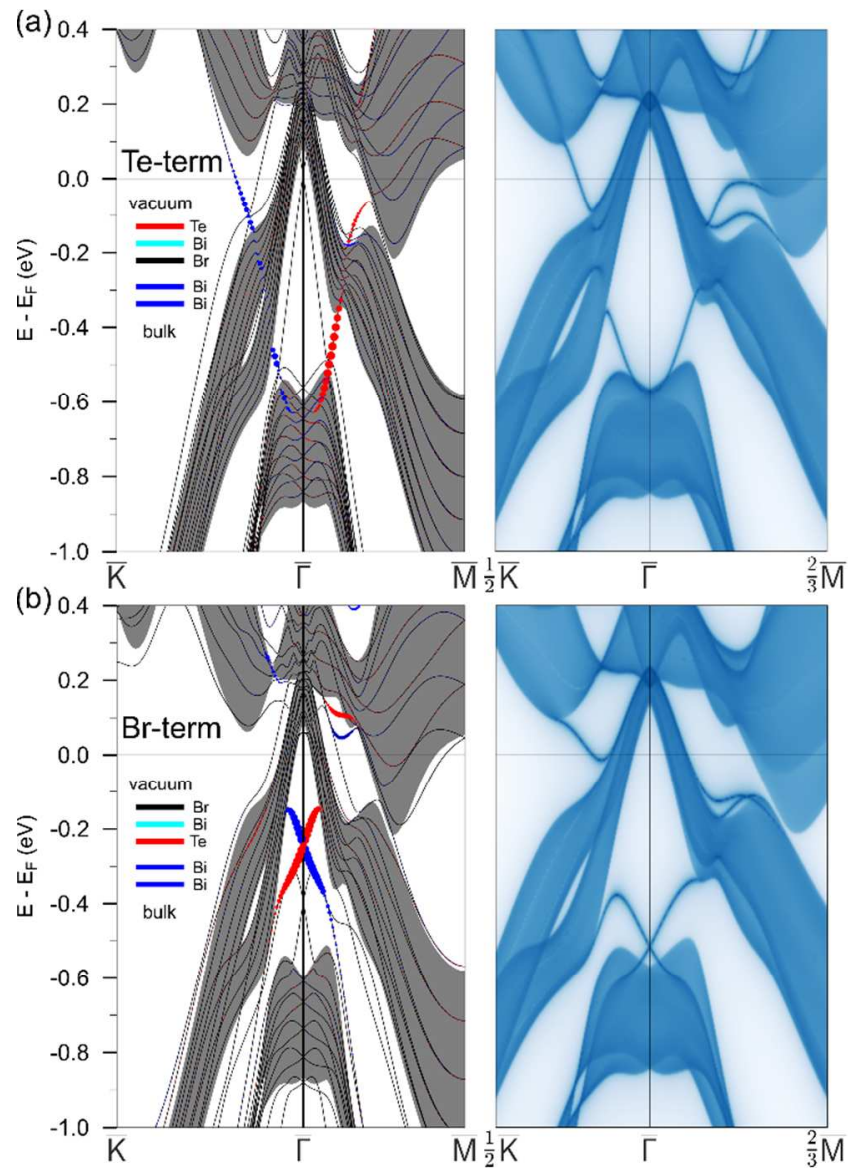

Fig. 11. Surface electronic structures of the Te- and Br-terminations of $\mathrm{Bi}_{3} \mathrm{TeBr}$. For surface states, the radii of the red and blue circles symbolize the values of the in-plane components of spin eigenvalues perpendicular to the shown direction of the $2 \mathrm{D}$ Brillouin zone. The results of DFT calculations are presented in the left column, the results of TB calculations - in the right column.

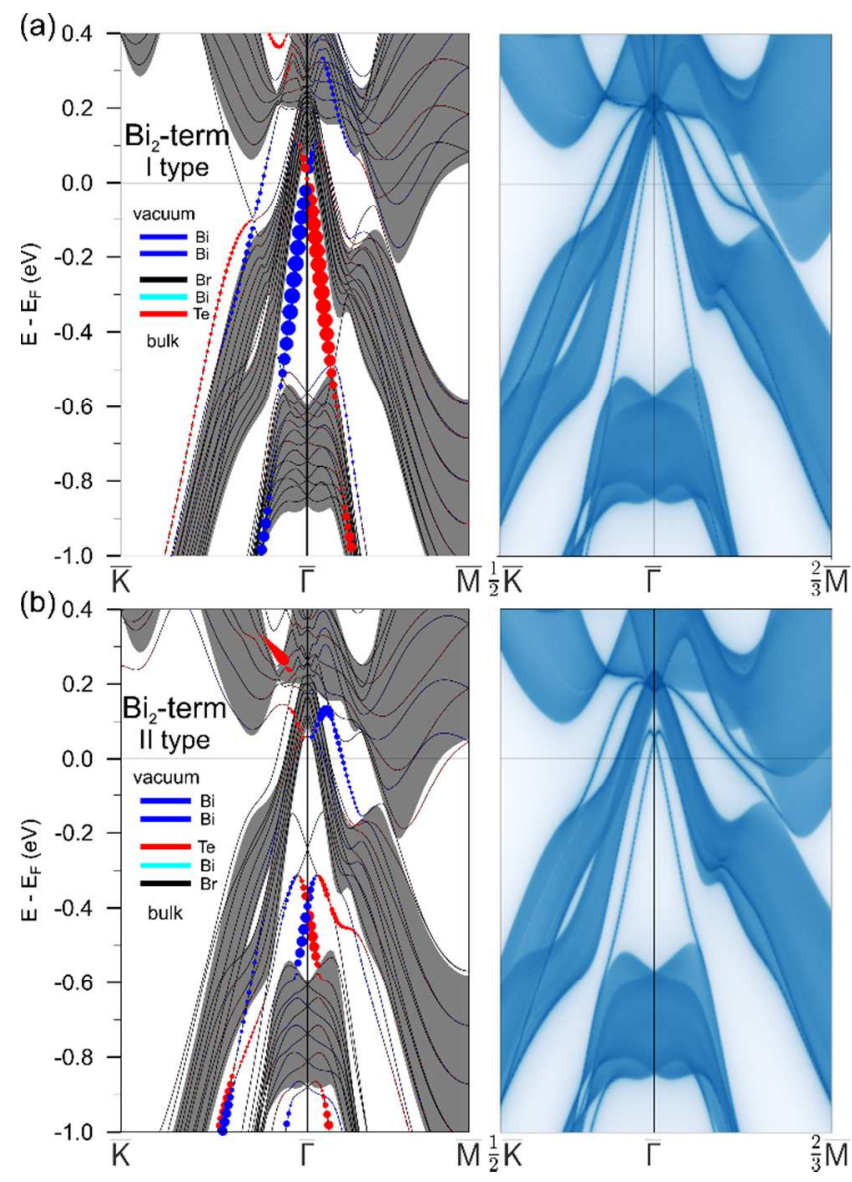

Fig. 12. Surface electronic structures of the two Bi2terminations (I and II type) of $\mathrm{Bi}_{3} \mathrm{TeBr}$. For surface states, the radii of the red and blue circles symbolize the values of the in-plane components of spin eigenvalues perpendicular to the shown direction of the 2D Brillouin zone. The results of DFT calculations are presented in the left column, the results of TB calculations - in the right column.

To conclude, the topological character of new tellurobromides coincides with the corresponding telluroiodides $^{33}$. $\mathrm{Bi}_{2} \mathrm{TeBr}$ is essentially a dual topological insulator, i. e. a topological crystalline insulator owing to the mirror planes and a weak $3 \mathrm{D}$ topological insulator with $Z_{2}=$ $\mathrm{o}$;(ool). The topological band gap in $\mathrm{Bi}_{2} \mathrm{TeBr}$ crosses the Fermi level almost in the full $3 \mathrm{D}$ BZ with an exception of a small electronic pocket along the $\Gamma-\mathrm{M}$ direction, that makes the electronic spectrum of $\mathrm{Bi}_{2} \mathrm{TeBr}$ semimetallic. $\mathrm{Bi}_{3} \mathrm{TeBr}$ is a topological metal with a complex hybridization of the states. A larger band gap is expected for elements with higher electronegativity, i. e. bromides vs. iodides, in the case of trivial insulators. It is, however, opposite in the topological regime due to the inverted (or negative) character of the gap. A transition between a topological and a trivial insulator proceeds via gap closing, and a topological semimetal may form at the boundary. Consequently, doping of topological insulators with lighter elements may cause the bulk band gap to close first and then to invert, as was observed for $\mathrm{TlBiSe}_{2-x} \mathrm{~S}_{x}$ and $\mathrm{Bi}_{2-x} \mathrm{In}_{x} \mathrm{Se}_{3} \cdot{ }^{70}$ Another example of this effect is a small- 
er gap in $\mathrm{Bi}_{2} \mathrm{Se}_{3}$ in comparison with $\mathrm{Bi}_{2} \mathrm{Te}_{3}$, as calculated within the GW-approach. ${ }^{71}$

\section{CONCLUSIONS}

Ternary bismuth tellurohalides $\mathrm{Bi}_{n} \mathrm{Te} X(X=\mathrm{I}, \mathrm{Br} ; n=1$, 2,3 ) constitute a structurally flexible materials' base for exploration of topological properties. Their structure and composition can be modified via incorporation of $\left[\mathrm{Bi}_{2}\right]$ modules and anionic substitutions, similarly to topologically non-trivial bismuth tellurides ${ }^{72}$. We have developed synthetic routes for bulk samples and crystals of $\mathrm{Bi}_{n} \mathrm{TeBr}$ $(n=2,3)$ layered heterostructures. Further stacking variants may exist as evidenced by the "intermediate" phase.

In many aspects, both structural and electronic, the discovered tellurobromides have appeared the same as the telluroiodides ${ }^{33}$. Ubiquitous stacking disorder, however, can be better resolved in the $\mathrm{Bi}_{n} \mathrm{TeBr}$ series, so that these compounds are most suitable for forthcoming studies of topological defects in the spirit of $[64,66]$. In contrast to a trivial insulator $\mathrm{BiTeBr}, \mathrm{Bi}_{2} \mathrm{TeBr}$ and $\mathrm{Bi}_{3} \mathrm{TeBr}$ are predicted to have topologically non-trivial electronic spectra by our calculations. They exemplify how enhanced chemical bonding along the stacking direction, exceeding van den Waals interactions, can drastically alter the electronic properties of the trivial insulating matrix of BiTeBr, which has a larger gap than BiTeI. In some sense, this is an alternative to "physical" pressure proposed to modify the properties of bulk BiTeI. ${ }^{14}$ Chemical bonding in $\mathrm{Bi}_{n} \mathrm{Te} X$ is realized through overlaps of the $p$-orbitals and structural/chemical similarity of the structure modules. For instance, $(\mathrm{BiTe})^{+}$is isoelectronic to $\mathrm{Bi}_{2}$ in terms of the valence electrons. ${ }^{59}$ This complementarity ensures the structural and compositional richness provided by various combinations of building units, i.e. the applicability of the modular approach to the crystal structures.

The modular view on $\mathrm{Bi}_{n} \mathrm{Te} X$ is not limited by structure aspects. The $\mathrm{Bi}_{n} \mathrm{Te} X$ lattices are not mere stacks of independent $2 \mathrm{D}$ TI modules, but instead realize a topological band inversion as a result of interlayer bonding. The ${ }_{\infty}^{2}\left[\mathrm{Bi}_{2}\right]$ module incorporated into the $\mathrm{BiTe} X$ matrix is not an ionic spacer, like bismuth-iodide fragments in $\mathrm{Bi}_{14} \mathrm{Rh}_{3} \mathrm{I}_{9}{ }^{73}$, but a rightful player in the game. Relatively low polarity of chemical bonding and highly diffuse $p$ orbitals of $\mathrm{Bi}$ support strong hybridization of the states between the adjacent modules along the stacking direction. Moreover, differences in the electronegativity between $\mathrm{I}$ and $\mathrm{Br}$ do not significantly affect the electronic properties of $\mathrm{Bi}_{n} \mathrm{Te} X$ with inverted topological gaps. The topological band inversion takes place between the states of two distinct structure fragments, ${ }_{\infty}^{2}\left[\mathrm{Bi}_{2}\right]$ and ${ }_{\infty}^{2}[\mathrm{BiTe} X]$, as found for $\mathrm{Bi}_{2} \mathrm{TeI}^{33}, \mathrm{Bi}_{2} \mathrm{TeBr}$ and $\mathrm{Bi}_{3} \mathrm{TeBr}$.

\section{EXPERIMENTAL SECTION}

Powder synthesis. Similar to $\mathrm{Bi}_{n} \mathrm{TeI}^{33}$, the target tellurobromides were synthesized from the stoichiometric mixtures of bismuth, tellurium and bismuth tribromide. Subsequent oxidation and occasional formation of $\mathrm{BiOBr}$ (transparent yellow, $T_{\mathrm{m}}=620{ }^{\circ} \mathrm{C}$ ) posed a much stronger issue for the bromides than for iodides. Additionally, high volatility of $\mathrm{BiBr}_{3}$ likely accounted for small deviations from the target compositions in some cases.

Polycrystalline samples were obtained by annealing of a stoichiometric mixture of $\mathrm{Bi}, \mathrm{Te}$, and $\mathrm{BiBr}_{3}$ (bismuth, MERCK, treated at $220{ }^{\circ} \mathrm{C}$ in $\mathrm{H}_{2}$-flow; tellurium, MERCK, $>99.9 \%$; bismuth(III)-bromide, synthesized from the elements and sublimated twice). The starting materials were pulverized by ball milling ("Pulverisette 23", Fritsch) for 25 minutes under argon atmosphere in a glovebox. The homogeneous powder was sealed in an evacuated silica ampoule. The samples of $\operatorname{Bi}_{n} \operatorname{TeBr}(n=2,3)$ were annealed at $400{ }^{\circ} \mathrm{C}$ and $385^{\circ} \mathrm{C}$, respectively, for at least three days.

Crystal Growth. Phase-pure powders synthesized via the above described procedure were used as batches for the crystal-growth experiments. Small single crystals (up to $200 \mu \mathrm{m}$ ) for single-crystal X-ray diffraction studies were obtained via mineralization for 2 weeks at 400 and $385^{\circ} \mathrm{C}$ for $\mathrm{Bi}_{2} \mathrm{TeBr}$ and $\mathrm{Bi}_{3} \mathrm{TeBr}$, respectively.

X-ray diffraction experiments. Single-crystal X-ray diffraction was measured on a four-circle Kappa APEX II CCD diffractometer (Bruker) with a graphite(0o2)monochromator and a CCD-detector at $T=296(2) \mathrm{K}$. Mo$\mathrm{K}_{\alpha}$ radiation $(\lambda=71.073 \mathrm{pm})$ was used. A numerical absorption correction based on an optimized crystal description ${ }^{74}$ was applied and the initial structure solution was performed in JANA2006 ${ }^{75}$. The structures were refined in SHELXL against $F_{\mathrm{o}}{ }^{2}{ }^{76,77}$ Further details on the crystal structure investigation of $\mathrm{BiTeBr}, \mathrm{Bi}_{2} \mathrm{TeBr}, \mathrm{Bi}_{3} \mathrm{TeBr}$ and $\mathrm{Bi}_{2} \mathrm{TeI}$ can be obtained from the Supporting Information (Tables S1-S9) and from the Fachinformationszentrum Karlsruhe, 76344 EggensteinLeopoldshafen, Germany (fax: (+49)7247-8o8-666; e-mail: crysdata@fiz-karlsruhe.de), on quoting the depository numbers CSD-434404, 434405, 434406, and 434407, respectively.

Powder X-ray diffraction data were measured using a X'Pert Pro diffractometer (PANalytical) with BraggBrentano geometry, a curved Ge(111) monochromator, and $\mathrm{Cu}-\mathrm{K} \alpha \mathbf{1}$ radiation $(\lambda=154.06 \mathrm{pm})$. Variable divergence slits were used to keep the illuminated sample area constant.

Graphics of the structures were developed with Diamond $^{78}$ software.

Thermal properties of $\mathrm{BiTeBr}, \mathrm{Bi}_{2} \mathrm{TeBr}$ and $\mathrm{Bi}_{3} \mathrm{TeBr}$ were analyzed by means of differential scanning calorimetry (DSC) using a Setaram Labsys ATD-DSC device with a k-probe $\left(\mathrm{Ni}-\mathrm{Cr} / \mathrm{Ni}-\mathrm{Al} ; \mathrm{T}_{\max }=800{ }^{\circ} \mathrm{C}\right)$ and $\mathrm{Al}_{2} \mathrm{O}_{3}$ as a reference compound. Heating and cooling rates of $2 \% \mathrm{~min}$ were employed. The substance was sealed in an evacuated silica glass ampoule.

Transmission and Scanning Electron Microscopy. SEM images were collected on a Hitachi SU8020 microscope. Acceleration voltages of 10 to $20 \mathrm{kV}$ and current of 
$10 \mu \mathrm{A}$ were used to create the SEM images. Energy dispersive X-ray spectra (EDX) were collected using an Oxford Silicon Drift X-Max ${ }^{\mathrm{N}}$ detector at an acceleration voltage of $20 \mathrm{kV}$ and a $100 \mathrm{~s}$ accumulation time. The EDX analysis was performed using the P/B-ZAF standardless method (where $\mathrm{Z}=$ atomic no. correction factor, $\mathrm{A}=$ absorption correction factor, $\mathrm{F}=$ fluorescence factor, $\mathrm{P} / \mathrm{B}=$ peak to background model).

High-resolution transmission electron microscopy (HRTEM) and selected area electron diffraction (SAED) studies were performed on a FEI Titan F2o microscope with CS-correction operating at $80 \mathrm{kV}$ and on a CM2O microscope (Philips) operating at $200 \mathrm{kV}$. For image acquisition, a $2 \mathrm{k} \times 2 \mathrm{k}$ Slow-Scan CCD-Camera (Gatan) was used. Image simulations were done with the JEMS software $^{79}$. Samples were ground in ethanol and deposited on a lacey carbon grid. In addition, thin lamellas were cut from a single crystal of $\mathrm{Bi}_{3} \mathrm{TeBr}$ and a crystal of the "intermediate" phase perpendicular to the largest facets, i.e. along the stacking direction, with an ultramicrotome (Ultracut, Leica Microsystems) equipped with a diamond knife. Prior to it, the crystals were embedded in the epoxy resin Epon (Fluka) that was then polymerized at $60{ }^{\circ} \mathrm{C}$. The nominal lamella thickness was approx. $50 \mathrm{~nm}$ while locally crystalline flakes with lower thickness were observed.

Computational details. Electronic structure calculations were carried out within the density functional theory using the projector augmented-wave method ${ }^{80}$ as implemented in the VASP $\operatorname{code}^{81,82} .11 \times 11 \times 1(7 \times 7 \times 1) k$ point mesh has been employed in the case of surface (bulk) calculations. The exchange-correlation energy was treated using the generalized gradient approximation ${ }^{83}$. Exchange-correlation functional Perdew-Becke-Ernzehof (PBE) was used. The Hamiltonian contained the scalar relativistic corrections and the spin-orbit coupling was treated by second variation method. The cut-off planewave basis was chosen to be $300 \mathrm{meV}$.

A repeated slab model was used to calculate the surface electronic structures of the title compounds. A vacuum gap of about $15 \AA$ was introduced between the slabs to prevent their interaction from both sides. 32 atomic planes were used to simulate one Te-terminated slab of $\mathrm{Bi}_{2} \mathrm{TeBr}$, and 30 atomic plane - to model a $\mathrm{Br}$-terminated slab of $\mathrm{Bi}_{2} \mathrm{TeBr}$. Thus, finite-size effects for surface states were prevented. 38 atomic layers were used to simulate the $[\mathrm{Te}-\mathrm{Bi}-\mathrm{Br} \cdots \mathrm{Bi}-\mathrm{Bi}]-$ and the $[\mathrm{Bi}-\mathrm{Bi} \cdots \mathrm{Br}-\mathrm{Bi}-\mathrm{Te}]-$ terminations in $\mathrm{Bi}_{3} \mathrm{TeBr}$. 30 atomic layers were used to simulate the $[\mathrm{Br}-\mathrm{Bi}-\mathrm{Te} \cdots \mathrm{Bi}-\mathrm{Bi}]-$ and the $[\mathrm{Bi}-\mathrm{Bi} \cdots \mathrm{Te}-\mathrm{Bi}-$ $\mathrm{Br}$-terminations in $\mathrm{Bi}_{3} \mathrm{TeBr}$.

The large slab Hamiltonian within tight-binding method derived from the bulk one was afterwards used to calculate surface Green functions. ${ }^{84-86}$ Hence the TB models were constructed using WANNIER9o code ${ }^{87,88}$. The chosen basis consisted of six spinor p-type orbitals for each atom: $\left|p_{x}^{\uparrow}\right\rangle,\left|p_{y}^{\uparrow}\right\rangle,\left|p_{z}^{\uparrow}\right\rangle,\left|p_{x}^{\downarrow}\right\rangle,\left|p_{y}^{\downarrow}\right\rangle,\left|p_{z}^{\downarrow}\right\rangle$. The low-lying sorbitals were not taken into consideration.

\section{ASSOCIATED CONTENT}

Supporting Information. PXRD of the synthesized samples, experimental DSC data and discussion, HRTEM data, unit cell parameters for $\mathrm{BiTeI}_{\mathrm{x}} \mathrm{Br}_{1-\mathrm{x}}$, additional crystallographic data (incl. atomic parameters, isotropic displacement parameters and selected interatomic distances for $\operatorname{Bi}_{n} \operatorname{TeBr}(n=1,2$, 3), bulk band structures without spin-orbit coupling are available free of charge via the Internet at http://pubs.acs.org.

\section{AUTHOR INFORMATION}

\section{Corresponding Author \\ *Email: anna.isaeva@tu-dresden.de}

\section{Author Contributions}

The manuscript was written through contributions of all authors. All authors have given approval to the final version of the manuscript.

\section{Funding Sources}

This work was supported by the German Research Foundation (DFG) in the framework of the Special Priority Program (SPP 1666) "Topological Insulators" and by the ERANETChemistry Program. We acknowledge support by Academic D. I. Mendeleev Fund Program of Tomsk State University (project No. 8.1.01.2018), by Saint Petersburg State University (project No. 15.61.202.2015), by Ministry of Education and Science of the Russian Federation within the framework of the governmental program "Megagrants" (State Task No. $3.8716 .2017 / \mathrm{P}_{220}$ or $\left.3.8716 .2017 / 9.10\right)$, by Russian Science Foundation No. 18-12-00169 (for surface electronic structure within tight-binding method), by Spanish Ministry of Science and Innovation (Grants No. FIS 2013-48286-Co2-02-P, No. FIS 2013-48286- Co2-440 01-P, and No. FIS 2016-75862-P).

\section{Notes}

The authors declare no competing financial interest.

\section{ACKNOWLEDGMENT}

This research received its initial impetus and supervision from Prof. Boris A. Popovkin (1937-2008), to whom the authors are indebted for inspiration. We are grateful to Prof. C. T. Koch, Dr. Wouter Van den Broek (Humboldt-University Berlin, Germany) and Prof. U. Kaiser (University of Ulm, Germany) for the provided TEM facilities at the Physics Department of the University of Ulm, and to Mr. E. Schmid (Central facility for electron microscopy, University of Ulm) for ultramicrotomy of our samples.

\section{ABBREVIATIONS}

TI topological insulator; DSC differential scanning calorimetry; PXRD powder X-ray diffraction; SCXRD single-crystal Xray diffraction; SEM scanning electron microscopy; TEM transmission electron microscopy; ED electron diffraction; HRTEM high-resolution transmission electron microscopy; SOC spin-orbit coupling; BZ Brillouin zone.

\section{REFERENCES}

(1) Kulbachinskii, V. A.; Kytin, V. G.; Lavrukhina, Z. V.; Kuznetsov, A. N.; Shevelkov, A. V. Galvanomagnetic and thermoe- 
lectric properties of $\mathrm{BiTeBr}$ and BiTeI single crystals and their electronic structure. Semiconductors 2010, 44, 1548-1553.

(2) Kulbachinskii, V. A.; Kytin, V. G.; Kudryashov, A. A.; Kuznetsov, A. N.; Shevelkov, A. V. On the electronic structure and thermoelectric properties of BiTeBr and BiTeI single crystals and of BiTeI with the addition of Bilz and CuI. J. Solid State Chem. 2012, 193, 154-160.

(3) Ishizaka, K.; Bahramy, M.S.; Murakawa, M.; Sakano, M.; Shimojima, T.; Sonobe, T.; Koizumi, K.; Shin, S.; Miyahara, M.; Kimura, A.; Miyamoto, M.; Okuda, M.T.; Namatame, H.; Taniguchi, M.; Arita, R.; Nagaosa, N.; Kobayashi, K.; Murakami, Y.; Kumai, R.; Kaneko, Y.; Onose, Y.; Tokura, T. Giant Rashba-type spin splitting in bulk BiTeI. Nat. Mater. 2011, 10, 521-526.

(4) Bahramy, M. S.; Arita, R.; Nagaosa. N. Origin of giant bulk Rashba splitting: Application to BiTeI. Phys. Rev. B 2011, 84, 041202.

(5) Eremeev, S. V.; Nechaev, I. A.; Koroteev, Yu. M.; Echenique, P. M.; Chulkov E. V. Ideal Two-Dimensional Electron Systems with a Giant Rashba-Type Spin Splitting in Real Materials: Surfaces of Bismuth Tellurohalides. Phys. Rev. Lett. 2012, 108, 246802 .

(6) Rusinov, I. P.; Nechaev, I. A.; Eremeev, S. V.; Friedrich, C.; Blügel, S.; Chulkov E. V. Many-body effects on the Rashba-type spin splitting in bulk bismuth tellurohalides. Phys. Rev. B 2013, 87, 205103.

(7) Sakano, M.; Miyawaki, J.; Chainani, A.; Takata, Y.; Sonobe, T.; Shimojima, T.; Oura, M.; Shin, S.; Bahramy, M. S.; Arita, R.; Nagaosa, N.; Murakawa, H.; Kaneko, Y.; Tokura, Y.; Ishizaka, K. Three-dimensional bulk band dispersion in polar BiTeI with giant Rashba-type spin splitting. Phys. Rev. B 2012, 86, 085204.

(8) Eremeev, S. V.; Nechaev, I. A.; Chulkov, E.V. Giant Rashbatype spin splitting at polar surfaces of BiTeI. JETP Letters 2012, $96,437-444$.

(9) Rusinov, I. P.; Tereshchenko, O. E.; Kokh, K. A.; Shakhmametova, A. R.; Azarov, I. A.; Chulkov, E. V. Role of anisotropy and spin-orbit interaction in the optical and dielectric properties of BiTeI and BiTeCl compounds. JETP Letters 2015, 101, 507-512.

(10) Sklyadneva, I. Yu.; Heid, R.; Bohnen, K.-P.; Chis, V.; Volodin, V. A.; Kokh, K. A.; Tereshchenko, O. E.; Echenique, P. M.; Chulkov, E. V. Lattice dynamics of bismuth tellurohalides. Phys. Rev. B 2012, 86, 094302.

(11) Crepaldi, A.; Moreschini, L.; Autès, G.; Tournier-Colletta, C.; Moser, S.; Virk, N.; Berger, H.; Bugnon, Ph.; Chang, Y. J.; Kern, K.; Bostwick, A.; Rotenberg, E.; Yazyev, O. V.; Grioni, M. Giant ambipolar Rashba effect in the semiconductor BiTeI. Phys. Rev. Lett. 2012, 109, 096803.

(12) Landolt, G.; Eremeev, S.V.; Koroteev, Yu.M.; Slomski, B.; Muff, S.; Neupert, T.; Kobayashi, M.; Strocov, V.N.; Schmitt, T.; Aliev, Z.S.; Babanly, M.B.; Amiraslanov, I.R.; Chulkov, E.V.; Osterwalder, J.; Dil, J.H. Disentanglement of surface and bulk Rashba spin splittings in noncentrosymmetric BiTeI. Phys. Rev. Lett. 2012, 109, 116403 .

(13) Landolt, G.; Eremeev, S. V.; Tereshchenko, O. E.; Muff, S.; Slomski, B.; Kokh, K.A.; Kobayashi, M.; Schmitt, T.; Strocov, V.N.; Osterwalder, J.; Chulkov, E. V.; Dil, J. H. Bulk and surface Rashba splitting in singe termination BiTeCl. New J. Phys. 2013, 15,085022 .

(14) Bahramy, M. S.; Yang, B.-J.; Arita, R.; Nagaosa, N. Emergence of non-centrosymmetric topological insulating phase in BiTeI under pressure. Nat. Commun. 2012, 3, 679.

(15) Chen, Y.; Xi, X.; Yim, W.-L.; Peng, F.; Wang, Y.; Wang, H.; Ma, Y.; Liu, G.; Sun, C.; Ma, C.; Chen, Z.; Berger, H. Highpressure phase transitions and structures of topological insulator BiTeI. J. Phys. Chem. C 2013, 117, 25677-25683.

(16) Rusinov, I. P.; Menshchikova, T. V.; Sklyadneva, I. Yu.; Heid, R.; Bohnen, K.-P.; Chulkov, E.V. Pressure effects on crystal and electronic structure of bismuth tellurohalides. New J. Phys. 2016, $18,113003$.

(17) Tran, M. K.; Levallois, J.; Lerch, P.; Teyssier, J.; Kuzmenko, A. B.; Autès, G.; Yazyev, O. V.; Ubaldini, A.; Giannini, E.; van der Marel, D.; Akrap, A. Infrared- and Raman-Spectroscopy Measurements of a Transition in the Crystal Structure and a Closing of the Energy Gap of BiTeI under Pressure. Phys. Rev. Lett. 2014, $112,047402$.

(18) Ideue, T.; Checkelsky, J. G.; Bahramy, M. S.; Murakawa, H.; Kaneko, Y.; Nagaosa, N.; Tokura, Y. Pressure variation of Rashba spin splitting toward topological transition in the polar semiconductor BiTeI. Phys. Rev. B 2014, 90, 161107.

(19) Sans, J. A.; Manjón, F. J.; Pereira, A. L. J.; Vilaplana, R.; Gomis, O.; Segura, A.; Muñoz, A.; Rodríguez-Hernández, P.; Popescu, C.; Drasar, C.; Ruleova, P. Structural, vibrational, and electrical study of compressed BiTeBr. Phys. Rev. B 2016, 93, 024110 .

(20) Park, J.; Jin, K.-H.; Jo, Y. J.; Choi, E.S.; Kang, W.; Kampert, E.; Rhyee, J.-S.; Jhi, S.-H.; Kim, J. S. Quantum Oscillation Signatures of Pressure-induced Topological Phase Transition in BiTeI. Sci. Rep. 2015, 5, 15973.

(21) Ohmura, A.; Higuchi, Y.; Ochiai, T.; Kanou, M.; Ishikawa, F.; Nakano, S.; Nakayama, A.; Yamada, Y.; Sasagawa, T. Pressureinduced topological phase transition in the polar semiconductor BiTeBr. Phys. Rev. B 2017, 95, 125203.

(22) Xi, X.; Ma, C.; Liu, Z.; Chen, Z.; Ku, W.; Berger, H.; Martin, C.; Tanner, D. B.; Carr, G. L. Signatures of a pressure-induced topological quantum phase transition in BiTeI. Phys. Rev. Lett. 2013, 111, 155701.

(23) Ponosov, Yu. S.; Kuznetsova, T. V.; Tereshchenko, O. E.; Kokh, K. A.; Chulkov, E. V. Dynamics of the BiTeI lattice at high pressures. JETP Lett. 2014, 98, 557-561.

(24) Ying, J. J.; Struzhkin, V. V.; Cao, Z. Y.; Goncharov, A. F.; Mao, H. K.; Chen, F.; Chen, X. H.; Gavriliuk, A. G.; Chen, X. J. Realization of insulating state and superconductivity in the Rashba semiconductor BiTeCl. Phys. Rev. B 2016, 93, 100504.

(25) Qi, Y.; Shi, W.; Naumov, P. G.; Kumar, N.; Sankar, R.; Schnelle, W.; Shekhar, C.; Chou, F. C.; Felser, C.; Yan, B.; Medvedev, S. A. Topological quantum phase transition and superconductivity induced by pressure in the bismuth tellurohalide BiTeI. Adv. Mater. 2017, 29, 1605965.

(26) VanGennep, D.; Linscheid, A.; Jackson, D. E.; Weir, S. T.; Vohra, Y. K.; Berger, H.; Stewart, G. R.; Hennig, R. G.; Hirschfeld, P. J.; Hamlin, J. J. Pressure-induced superconductivity in the giant Rashba system BiTeI. J. Phys. Condens. Matter 2017, 29, o9LTo2.

(27) Wu, L.; Yang, J.; Chi, M.; Wang, S.; Wie, P.; Zhang, W.; Chen, L.; Yang, J. Enhanced thermoelectric performance in $\mathrm{Cu}$ intercalated BiTeI by compensation weakening induced mobility improvement. Sci. Rep. 2015, 5, 14319.

(28) Wu, L.; Yang, J.; Zhang, T.; Wang, S.; Wei, P.; Zhang, W.; Chen, L.; Yang, J. Enhanced thermoelectric performance in the Rashba semiconductor BiTeI through band gap engineering. $J$. Phys. Condens. Matter 2016, 28, 085801.

(29) Kulbachinskii, V. A.; Kytin, V. G.; Lavrukhina, Z. V.; Kuznetsov, A. N.; Markelov, A. V.; Shevelkov, A. V. Thermoelectric Properties of BiTeI with Addition of $\mathrm{BiI}_{3}, \mathrm{CuI}$, and Overstoichiometric Bi. Semiconductors 2o11, 45, 845-849.

(30) Klimovskikh, I.I.; Shikin, A.M.; Otrokov, M.M.; Ernst, A.; Rusinov, I.P.; Tereshchenko, O.E.; Golyashov, V.A.; SanchezBarriga, J.; Varykhalov, A. Yu.; Rader, O.; Kokh, K.A.; Chulkov, E.V. Giant Magnetic Band Gap in the Rashba-Split Surface State of Vanadium-Doped BiTeI: A Combined Photoemission and $A b$ Initio Study. Sci. Rep. 2017, 7, 3353.

(31) Tu, K.; Wei, P.; Zhou, H.; Mu, X.; Zhu, W.; Nie, X.; Zhao, W. A thermoelectric performance study of layered BizTeI weak topological insulator materials. Energies 2018, 11, 891. 
(32) Savilov, S. V.; Khrustalev, V. N.; Kuznetsov, A. N.; Popovkin, B. A.; Antipin, M. Yu. New subvalent bismuth telluroiodides incorporating Bi2 layers: the crystal and electronic structure of BizTeI. Russ. Chem. Bull. 2005, 54, 87-92.

(33) Zeugner, A.; Kaiser, M.; Schmidt, P.; Menshchikova, T.V.; Rusinov, I.P.; Markelov, A.V.; Van den Broek, W.; Chulkov, E.V.; Doert, T.; Ruck, M.; Isaeva, A. Modular design with $2 \mathrm{D}$ topological-insulator building blocks: optimized synthesis and crystal growth, crystal and electronic structures of $\mathrm{Bi}_{\mathrm{x}} \mathrm{TeI}(\mathrm{x}=\mathbf{2}$, 3). Chem. Mater. 2017, 29, 1321-1337.

(34) Murakami, S. Quantum spin Hall effect and enhanced magnetic response by spin-orbit coupling. Phys. Rev. Lett. 2006, 97, 236805.

(35) Wada, M.; Murakami, S.; Freimuth, F.; Bihlmayer, G. Localized edge states in two-dimensional topological insulators: Ultrathin Bi films. Phys. Rev. B 2011, 83, 121310.

(36) Drozdov, I.K.; Alexandradinata, A.; Jeon, S.; Nadj-Perge, S.; Ji, H.; Cava, R.J.; Bernevig, B.A.; Yazdani, A. One-dimensional topological edge states of bismuth bilayers. Nat. Phys. 2014, 10, 664-669.

(37) Ohtsubo, Y.; Perfetti, L.; Goerbig, M. O.; Fevre, P. L.; Bertran, F.; Taleb-Ibrahimi, A. Non-trivial surface-band dispersion on Bi(111). New J. Phys. 2013, 15, 033041.

(38) Perfetti, L.; Faure, J.; Papalazarou, E.; Mauchain, J.; Marsi, M.; Goerbig, M. O.; Taleb-Ibrahimi, A.; Ohtsubo, Y. New aspects of electronic excitations at the bismuth surface: topology, thermalization and coupling to coherent phonons. J. Electron Spectrosc. Relat. Phenom. 2015, 201, 60-65.

(39) Ning, W.; Kong, F.; Xi, C.; Graf, D.; Du, H.; Han, Y.; Yang, J.; Yang, K.; Tian, M.; Zhang, Y. Evidence of topological twodimensional metallic surface states in thin bismuth nanoribbons. ACS Nano 2014, 8, 7506-7512.

(40) Ning, W.; Kong, F.; Han, Y.; Du, H.; Yang, J.; Tian, M.; Zhang, Y. Robust surface state transport in thin bismuth nanoribbons. Sci. Rep. 2014, 4, 7086.

(41) Ito, S.; Feng, B.; Arita, M.; Takayama, A.; Liu, R.-Y.; Someya, T.; Chen, W.-C.; Iimori, T.; Namatame, H.; Taniguchi, M.; Cheng, C.-M.; Tang, S.-J.; Komori, F.; Kobayashi, K.; Chiang, T.C.; Matsuda, I. Proving nontrivial topology of pure bismuth by quantum confinement. Phys. Rev. Lett. 2016, 117, 236402.

(42) Tang, P.; Yan, B.; Cao, W.; Wu, S.-C.; Felser, C.; Duan, W. Weak topological insulators induced by the interlayer coupling: A first-principles study of stacked $\mathrm{Bi}_{2}$ TeI. Phys. Rev. B 2014, 89, 041409(R).

(43) Rusinov, I.P.; Menshchikova, T.V.; Isaeva, A.; Eremeev, S.V.; Koroteev, Y.M.; Vergniory, M.G.; Echenique, P.M.; Chulkov, E.V. Mirror-symmetry protected non-TRIM surface state in the weak topological insulator $\mathrm{Bi}_{2}$ TeI. Sci Rep. 2016, 6, 20734 .

(44) Avraham, N.; Norris, A.; Sun, Y.; Qi, Y.; Pan, L.; Isaeva, A.; Zeugner, A.; Felser, C.; Yan, B.; Beidenkopf, H. Coexisting surface states in the weak and crystalline topological insulator $\mathrm{Bi}_{2} \mathrm{TeI}$. Available online at arxiv.org: 1708.09062.

(45) Isaeva, A.; Rasche, B.; Ruck, M. Bismuth-based candidates for topological insulators: Chemistry beyond BizTez. Phys. Status Solidi RRL, 2013, 7, 39-49.

(46) Hasan, M.Z.; Kane, C.L. Colloquium: Topological insulators. Rev. Mod. Phys. 2010, 82, 3045.

(47) Bradlyn, B.; Elcoro, L.; Cano, J.; Vergniory, M. G.; Wang, Z.; Felser, C.; Aroyo, M.I.; Bernevig, B.A. Topological quantum chemistry. Nature 2017, 547, 298-305.

(48) Takada, K.; Sakurai, H.; Takayama-Muromachi, E.; Izumi, F.; Dilanian, R. A.; Sasaki, T. Superconductivity in twodimensional $\mathrm{CoO}_{2}$ layers. Nature 2003, 422, 53-55.

(49) Terasaki, I.; Iwakawa, M.; Nakano, T.; Tsukuda, A.; Kobayashi, W. Novel thermoelectric properties of complex transitionmetal oxides. Dalton Trans. 2010, 39, 1005-1011.
(50) Kimber, S. A.; Kreyssig, A.; Zhang, Y.-Z.; Jeschke, H. O.; Valenti, R.; Yokaichiya, F.; Colombier, E.; Yan, J.; Hansen, T.C.; Chatterij, T.; McQueeney, R. J.; Canfield, P. C.; Goldman, A. I.; Argyriou, D. N. Similarities between structural distortions under pressure and chemical pressure in superconducting BaFezAs2. Nat. Mater. 2009, 8, 471-475.

(51) Dönges, E. Über Tellurohalogenide des dreiwertigen Antimons und Wismuts und über Antimon-und Wismut (III)tellurid und Wismut(III)-Selenid. Z. Anorg. Allg. Chem. 1951, 265, 56-61.

(52) Onopko, L.V.; Onopko, V.V.; Chepur, D.V.; Dovgoshei, N.I.; Turyanica, I.D.; Zayachkovskaya, N. F. Physical properties of BiTeI and BiTeBr. Neorg. Mater. 1974, 10, 1137-1138.

(53) Onopko, L.V.; Turyanica, I.D.; Onopko, V.V.; Chepur, D.V.; Zayachkovskaya, N. F.; Gryadil, I.A. In: Physics and Technology of Thin Films of Complex Semiconductors. Uzhgorod State University, 1972.

(54) Petasch, U.; Oppermann, H. Untersuchungen zum quasibinären System Bi2Tez/BiBr3. Z. Naturforsch. 1999, $54 b$, 487-490.

(55) Biltz, W. Raumchemie der festen Stoffe. Leopold Voss, Leipzig, 1934.

(56) von Schnering, H. G.; von Benda, H.; Kalveram, Ch. Wismutmonoiodid BiI, eine Verbindung mit $\mathrm{Bi}(\mathrm{o})$ und $\mathrm{Bi}(\mathrm{II}) . \mathrm{Z}$. Anorg. Allg. Chem. 1978, 438, 37-52.

(57) von Benda, H.; Simon, A.; Bauhofer, W. Zur Kenntnis von $\mathrm{BiBr}$ and $\mathrm{BiBr}_{1.167}$. Z. Anorg. Allg. Chem. 1978, 438, 53-67.

(58) Dikarev, E. V.; Popovkin, B. A.; Shevelkov, A. V. New polymolecular bismuth monohalides. Synthesis and crystal structures of $\mathrm{Bi}_{4} \mathrm{Br}_{\mathrm{x}} \mathrm{I}_{4-\mathrm{x}}(\mathrm{x}=1,2$, or 3). Russ. Chem. Bull. Int. Ed. 2oo1, 50, 2304-2309.

(59) Shevelkov, A.V.; Dikarev, E.V.; Shpanchenko, R.V.; Popovkin. B.A. Crystal structures of bismuth tellurohalides $\operatorname{BiTeX}(\mathrm{X}=\mathrm{Cl}, \mathrm{Br}, \mathrm{I})$ from X-ray powder diffraction data. J. Solid State. Chem. 1995, 114, 379-384.

(6o) Eremeev, S. V.; Rusinov, I. P.; Nechaev, I. A.; Chulkov, E.V. Rashba surface split surface states in BiTeBr. New. J. Phys. 2013, 15, 075015 .

(61) Sakano, M.; Bahramy, M.S.; Katayama, A.; Shimojima, T.; Murakawa, H.; Kaneko, Y.; Malaeb, W.; Shin, S.; Ono, K.; Kumigashira, H.; Arita, R.; Nagaosa, N.; Hwang, H.Y.; Tokura, Y.; Ishizaka, K. Strongly spin-orbit coupled two-dimensional electron gas emerging near the surface of polar semiconductors. Phys. Rev. Lett. 2013, 110, 107204.

(62) Akrap, A.; Teyssier, J.; Magrez, A.; Bugnon, P.; Berger, H.; Kuzmenko, A. B.; van der Marel, D. Optical properties of BiTeBr and BiTeCl. Phys. Rev. B 2014, 90, 035201.

(63) Cenzual, K.; Gelato, L. M.; Penzo, M.; Parthe, E. Overlooked symmetry in structures reported with monoclinic centred Bravais lattices; trigonal description of $\mathrm{Li}_{8} \mathrm{~Pb}_{3}, \mathrm{PtTe}_{2} \mathrm{Pt}_{3} \mathrm{Te}_{4}, \mathrm{Pt}_{2} \mathrm{Te}_{3}$, $\mathrm{LiFe}_{6} \mathrm{Ge}_{4}, \mathrm{LiFe}_{6} \mathrm{Ge}_{5}, \mathrm{CaGe}_{6} \mathrm{Te} 10$ and La3.266Mn1.1S6. Z. Kristallogr. 1990, 193, 217-242.

(64) Nechaev, I. A.; Eremeev, S. V.; Krasovskii, E. E.; Echenique, P. M.; Chulkov, E. V. Quantum spin Hall insulators in centrosymmetric thin films composed from topologically trivial BiTeI trilayers. Sci. Rep.2017, 7, 43666.

(65) Fiedler, S.; Bathon, Th.; Eremeev, S. V.; Tereshchenko, O.V.; Kokh, K.A.; Chulkov, E.V.; Sessi, P.; Bentmann, H.; Bode, M.; Reinert, F. Termination-dependent surface properties in the giant-Rashba semiconductors BiTeX $(\mathrm{X}=\mathrm{Cl}, \mathrm{Br}, \mathrm{I})$. Phys. Rev. $B$ 2015, 92, 235430.

(66) Eremeev, S. V.; Nechaev, I. A.; Chulkov, E. V. Two- and three-dimensional topological phases in BiTeX compounds. Phys. Rev. B 2017, 96, 155309.

(67) Fiedler, S.; El-Kareh, L.; Eremeev, S. V.; Tereshchenko, O. E.; Seibel, C.; Lutz, P.; Kokh, K. A.; Chulkov, E. V.; Kuznetsova, T. V.; Grebennikov, V. I.; Bentmann, H.; Bode, M.; Reinert, F. De- 
fect and structural imperfection effects on the electronic properties of BiTeI surfaces. New J. Phys. 2014, 16, 075013.

(68) Tournier-Colletta, C.; Autes, G.; Kierren, B.; Bugnon, Ph.; Berger, H.; Fagot-Revurat, Y.; Yazyev, O. V.; Grioni, M.; Malterre, D. Atomic and electronic structure of a Rashba p-n junction at the BiTeI surface. Phys. Rev. B 2014, 89, 085402.

(69) Butler, C. J.; Yang, H.-H.; Hong, J.-Y.; Hsu, S.-H.; Sankar, R.; Lu, C.-I.; Lu, H.-Y.; Yang, K.-H. O.; Shiu, H.-W.; Chen, C.-H.; Kaun, C.-C.; Shu, G.-J.; Chou, F.-C.; Lin, M.-T. Mapping polarization induced surface band bending on the Rashba semiconductor BiTeI. Nat. Commun. 2014, 5, 4066.

(70) Armitage, N. P.; Mele, E. J.; Vishwanath, A. Weyl and Dirac semimetals in three-dimensional solids. Rev. Mod. Phys. 2018, 90, 015001.

(71) Aguilera, I.; Friedrich, Ch.; Bihlmayer, G.; Blügel, S. GW study of topological insulators $\mathrm{Bi}_{2} \mathrm{Se}_{3}, \mathrm{Bi}_{2} \mathrm{Te}_{3}$ and $\mathrm{Sb}_{2} \mathrm{Te}$ : Beyond the perturbative one-shot approach. Phys. Rev. B 2013, 88, 045206.

(72) Cava, R. J.; Ji, H.; Fuccillo, M. K.; Gibson, Q. D.; Hor, Y. S. Crystal Structure and Chemistry of Topological Insulators. J. Mater. Chem. C 2013, 1, 3176-3189.

(73) Rasche, B.; Isaeva, A.; Ruck, M.; Borisenko, S.; Zabolotnyy, V.; Büchner, B.; Koepernik, K.; Ortix, C.; Richter, M.; van den Brink, J. Stacked topological insulator built from bismuth-based graphene sheet analogues. Nat. Mater. 2013, 12, 422-425.

(74) X-Shape, Crystal Optimization for Numerical Absorption Correction Program. v. 2.12.2, Stoe \& Cie GmbH, Darmstadt, 2009.

(75) Petricek, V.; Dusek, M.; Palatinus, L. JANAzoo6, The Crystallographic Computing System. Institute of Physics, Praha, Czech Republic, 2011.

(76) Sheldrick, G. M. SHELXL, Program for Crystal Structure Refinement - Multi-CPU v. 2014/7, Georg-August-Universität Göttingen, Göttingen, Germany, 2014.
(77) Sheldrick, G. M. A short history of SHELX. Acta Crystallogr., Sect. A: Found. Crystallogr. 2008, 64, 112-122.

(78) Brandenburg, K. Diamond 3.2k, Crystal Impact GbR, Bonn, 2014.

(79) Stadelmann, P. JEMS software, v. 4.2731U2015, http://cimewww.epfl.ch/people/stadelmann/jemsWebSite/jems. htlm, 2012.

(8o) Blöchl, P. E. Projector augmented-wave method. Phys. Rev. $B$ 1994, 50, 17953-17979.

(81) Kresse, G.; Furthmüller, J. Efficient iterative schemes for ab initio total-energy calculations using a plane-wave basis set. Phys. Rev. B 1996, 54, 11169-11186.

(82) Kresse, G.; Joubert, D. From ultrasoft pseudopotentials to the projector augmented-wave method. Phys. Rev. B 1999, 59, 1758-1775.

(83) Perdew, J. P.; Burke, K.; Ernzerhof, M. Generalized gradient approximation made simple. Phys. Rev. Lett. 1996, 77, 38653868 .

(84) Lopez Sancho, M. P.; Lopez Sancho, J. M.; Rubio, J. J. Quick iterative scheme for the calculation of transfer matrices: application to Mo(100). J. Phys. F: Met. Phys. 1984, 14, 1205-1215.

(85) Lopez Sancho, M. P.; Lopez Sancho, J. M.; Rubio, J. J. Highly convergent schemes for the calculation of bulk and surface Green functions. J. Phys. F: Met. Phys. 1985, 15, 851-858.

(86) Henk, J.; Schattke, W. A subroutine package for computing Green's functions of relaxed surfaces by the renormalization method. Comput. Phys. Commun. 1993, 77, 69-83.

(87) Marzari, N.; Vanderbilt D. Maximally localized generalized Wannier functions for composite energy bands. Phys. Rev. B 1997, 56, 12847.

(88) Zhang, W.; Yu, R.; Zhang, H.-J.; Dai, X.; Fang, Z. Firstprinciples studies of the three-dimensional strong topological insulators BizTe3, Bi2Sez and Sb2Te3. New J. Phys. 2010, 12, 065013. 
Insert Table of Contents artwork here

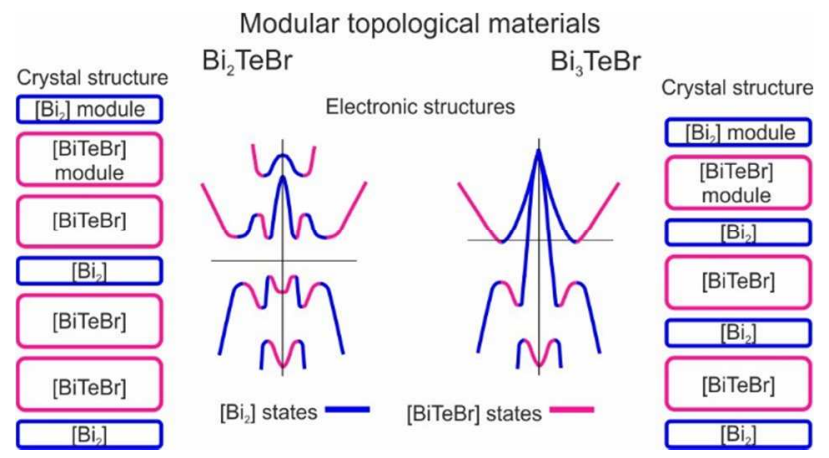

